Supplementary Information for

\title{
Transboundary environmental footprints of urban food supply chain and mitigation strategies
}

Yuanchao $\mathrm{Hu}^{1,2,3}$, Shenghui Cui1 ${ }^{1,3,}$, Xuemei Bai ${ }^{4, *}$, Yong-Guan $\mathrm{Zhu}^{1,3}$, Bing $\mathrm{Gao}^{1,3}$, Anu

Ramaswami $^{5}$, Jianxiong Tang ${ }^{1,2,3}$, Miaohong Yang ${ }^{1,6}$, Qianhu Zhang ${ }^{1,2,3}$, Yunfeng Huang ${ }^{7}$

1. Key Laboratory of Urban Environment and Health, Institute of Urban Environment, Chinese Academy of Sciences, Xiamen 361021, China

2. University of Chinese Academy of Sciences, No.19(A) Yuquan Road, Beijing 100049, China

3. Xiamen Key Lab of Urban Metabolism, Xiamen 361021, China

4. Fenner School of Environment and Society, Australian National University, Canberra ACT 0200, Australia

5. Hubert H. Humphrey School of Public Affairs, University of Minnesota, Minneapolis, Minnesota 55455, United States

6. Coastal and Ocean Management Institute, Xiamen University, Xiamen 361102, China

7. College of Food and Biotechnology, Jimei University, Xiamen 361024, China

*Email: shcui@iue.ac.cn; xuemei.bai@anu.edu.au

\section{Contents}

Transboundary environmental footprints of urban food supply chain and mitigation strategies .... S1

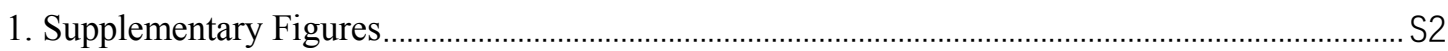

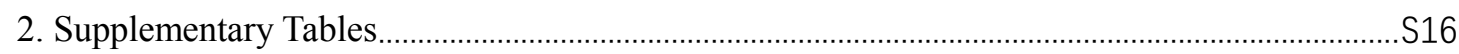

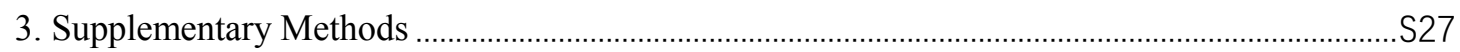

3.1. Environmental accounts of agriculture and other sectors................................................S27

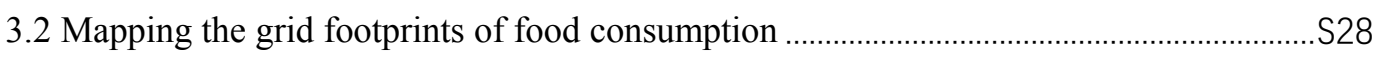

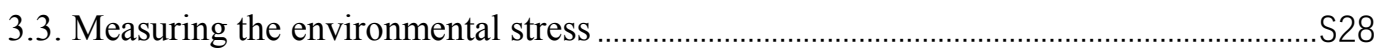

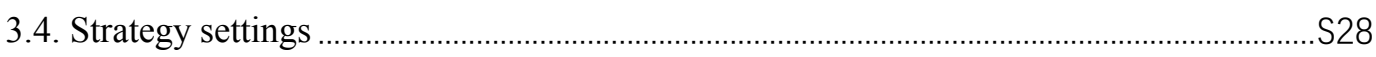

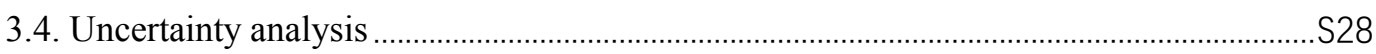

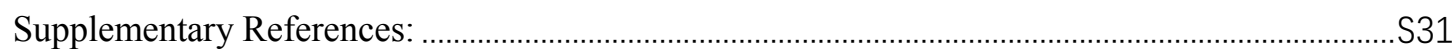




\section{Supplementary Figures}
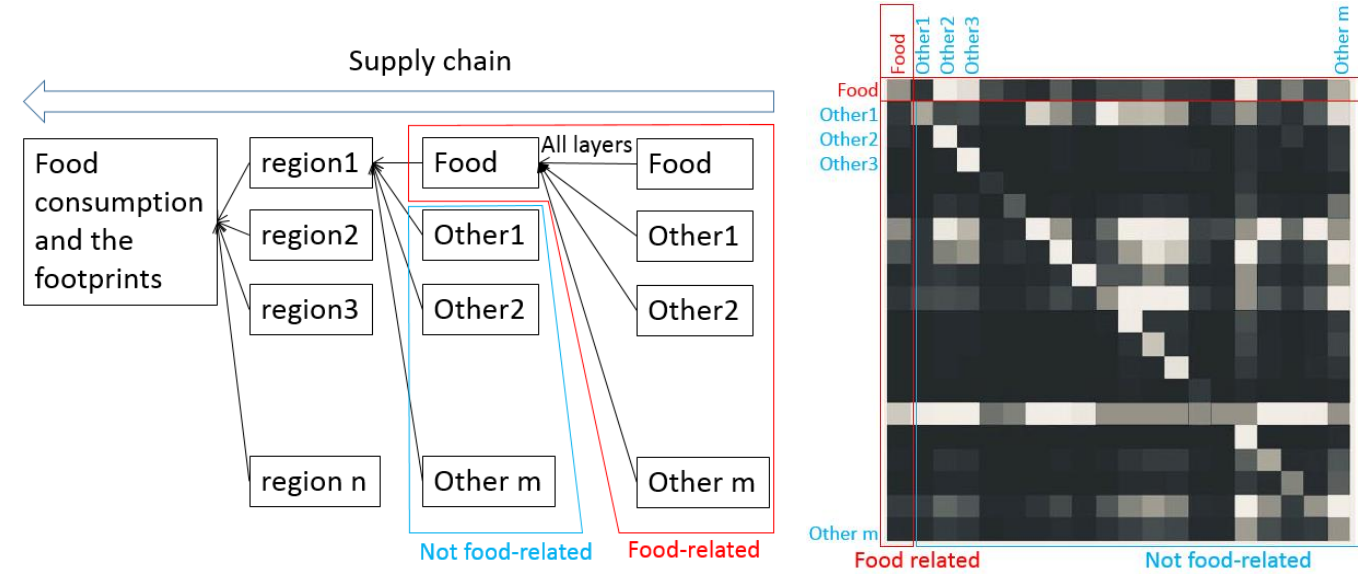

Supplementary Figure 1. The definition of 'food-related' sectors. 
a

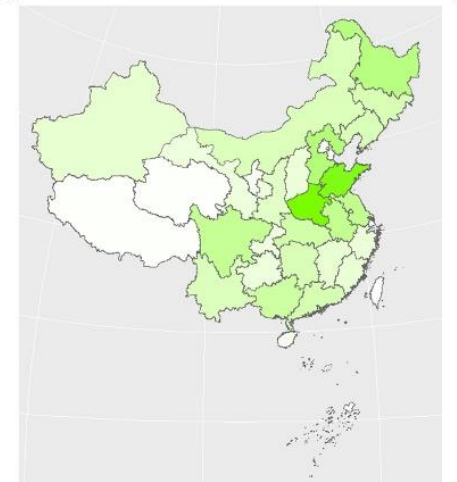

d

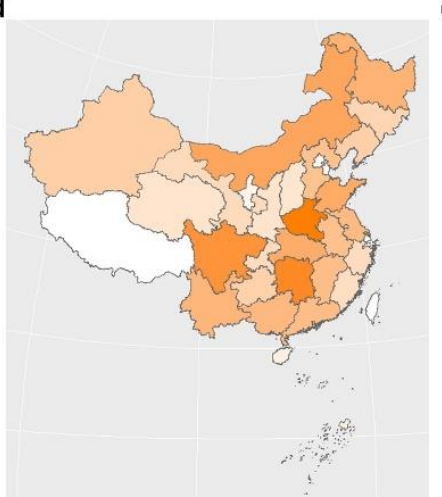

b
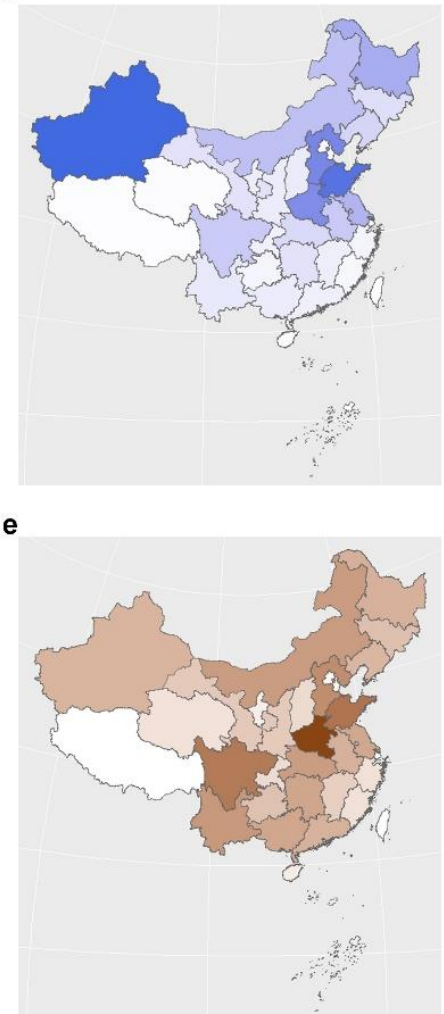

C

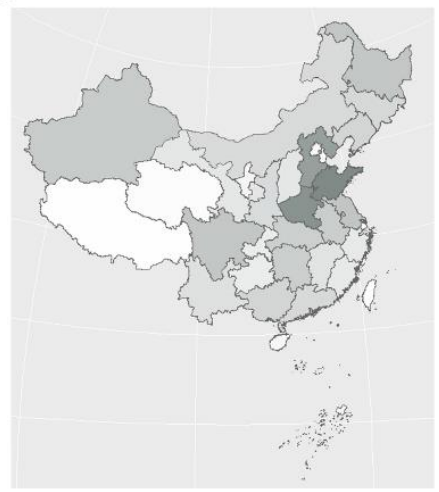

f

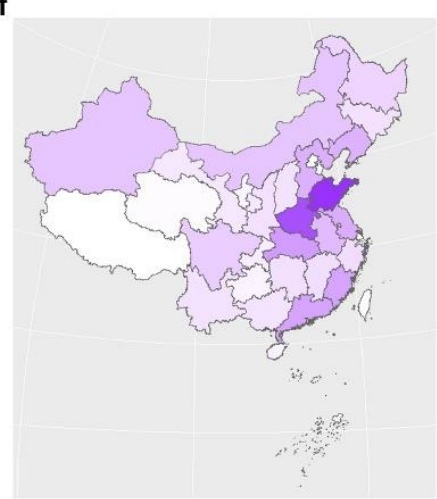

Supplementary Figure 2. Direct environmental impacts of food production in Chinese provinces. a. green water, in million $\mathrm{m}^{3} ; \mathbf{b}$. blue water, in million $\mathrm{m}^{3}$; c. grey water, in million $\mathrm{m}^{3}$; d. carbon emission, in million tons; e. reactive nitrogen emission, in ktN; f. phosphorus emission, in ktP. The darker color means larger impact in that region, while the lighter color means smaller impact in that region. 


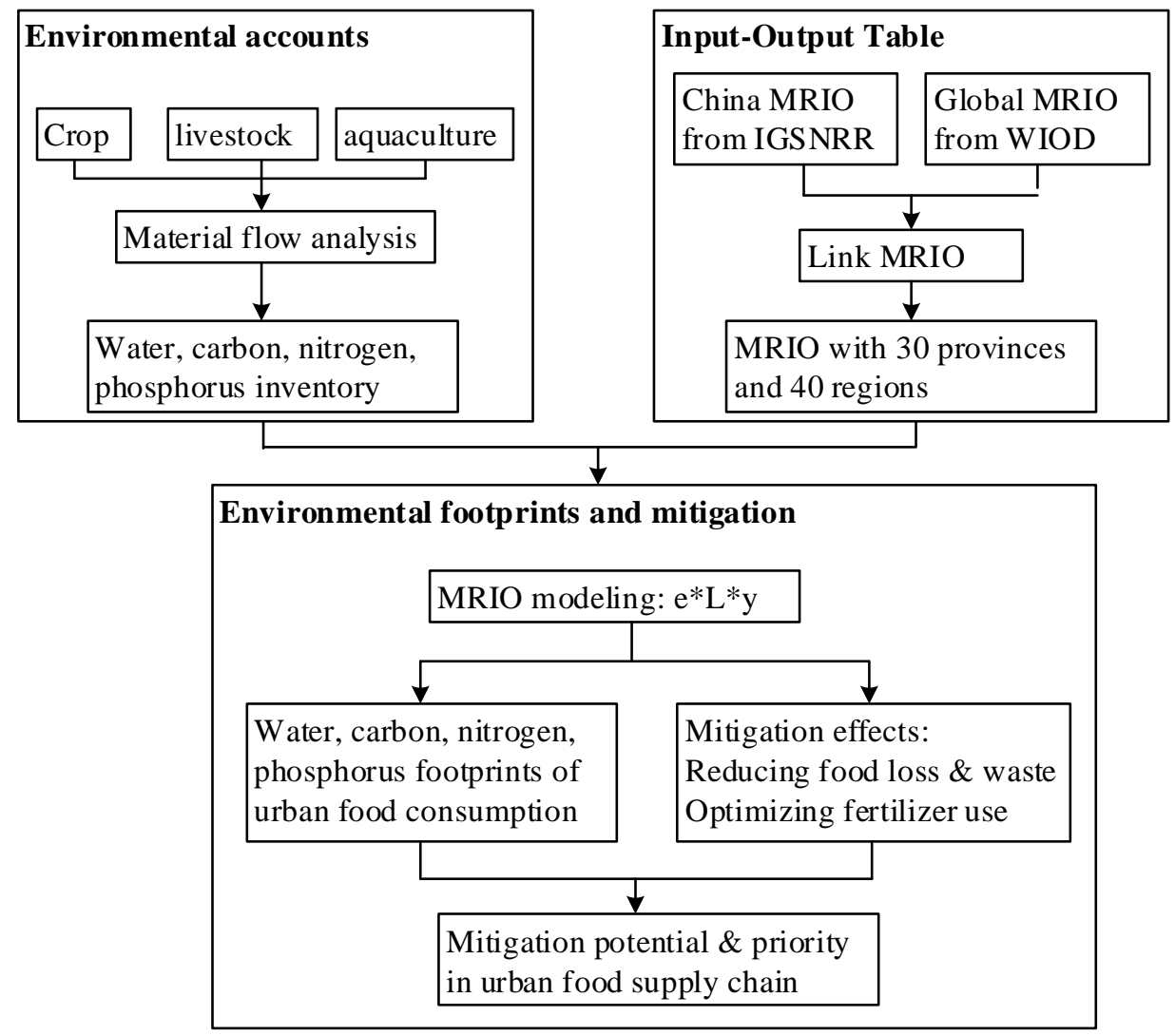

Supplementary Figure 3. Systematical framework of this study. 


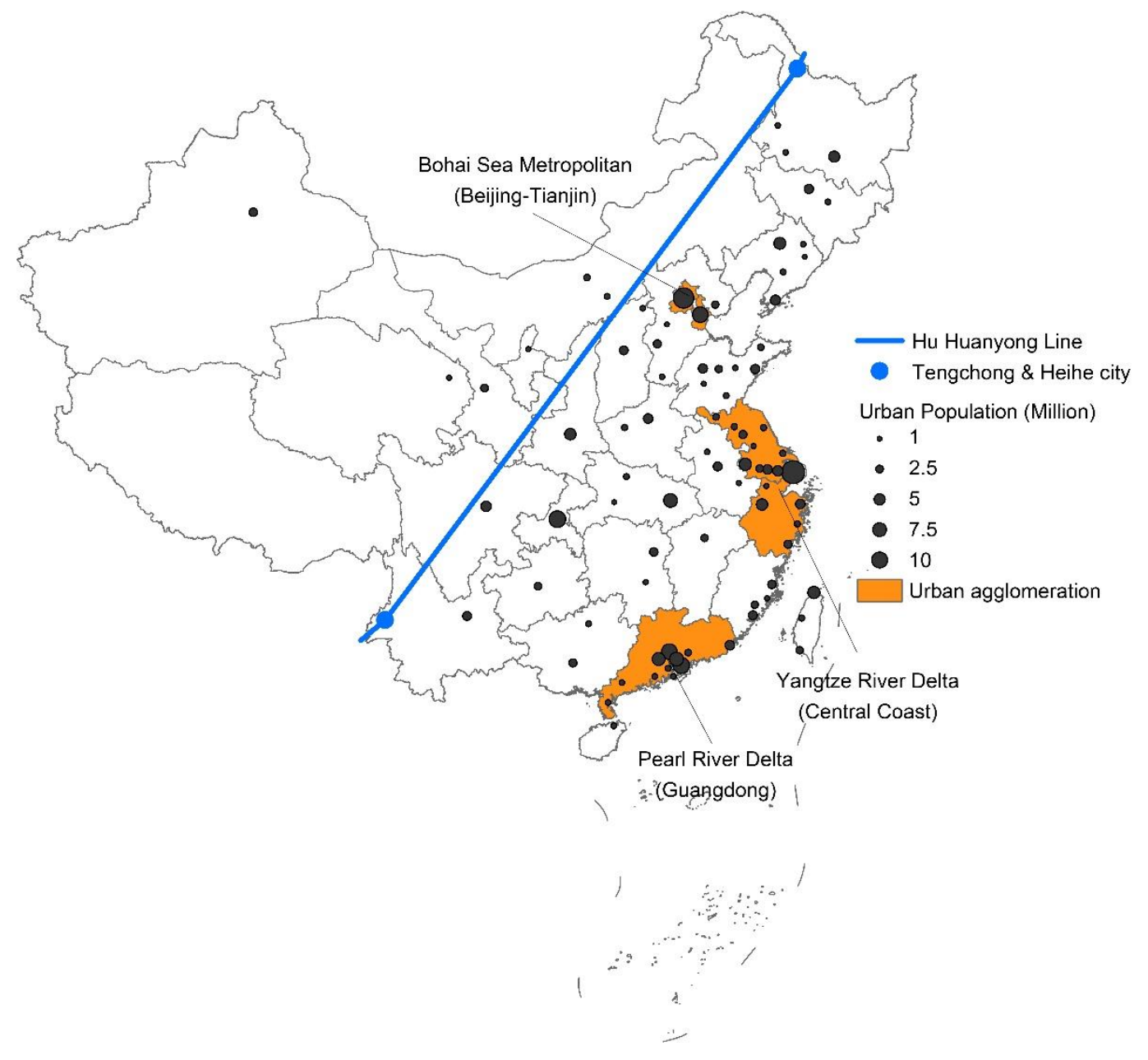

Supplementary Figure 4. Distribution of Chinese cities with urban population larger than one million people. The three major urban regions are Jing-Jin metropolitan (include Beijing and Tianjin city), Yangtze River Delta (include Shanghai, Zhejiang and Jiangsu province) and Pearl River Delta (Guangdong province). Unit is in million people. 


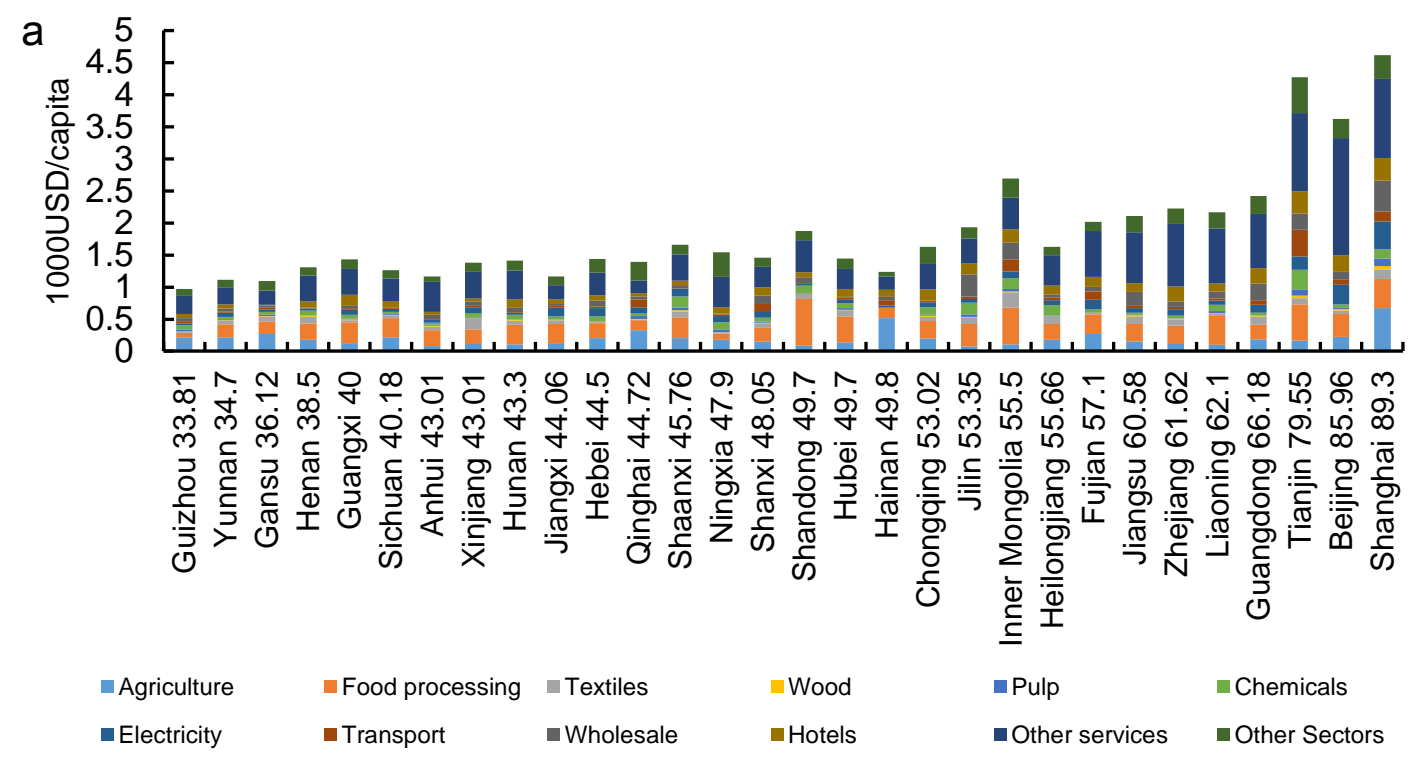

b

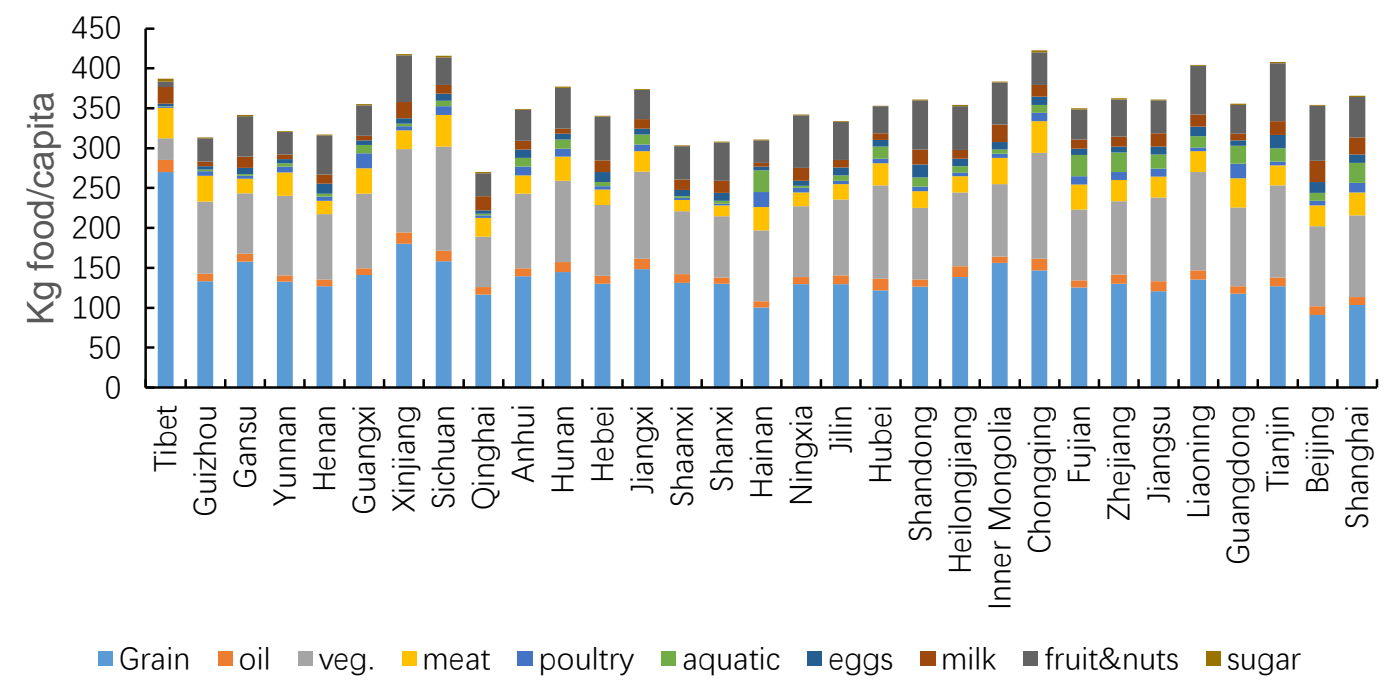

Supplementary Figure 5. Regional household final consumption level versus increasing urbanization rate in 2010. A) per capita final consumption expenditure by households in 2010, data from multi-regional input-output tables ${ }^{1}$, values are in US $\$ 1000$ /cap. B) per capita household food consumption in 2015 from National Bureau of Statistics of China ${ }^{2}$, values are in $\mathrm{kg}$ food/cap. 


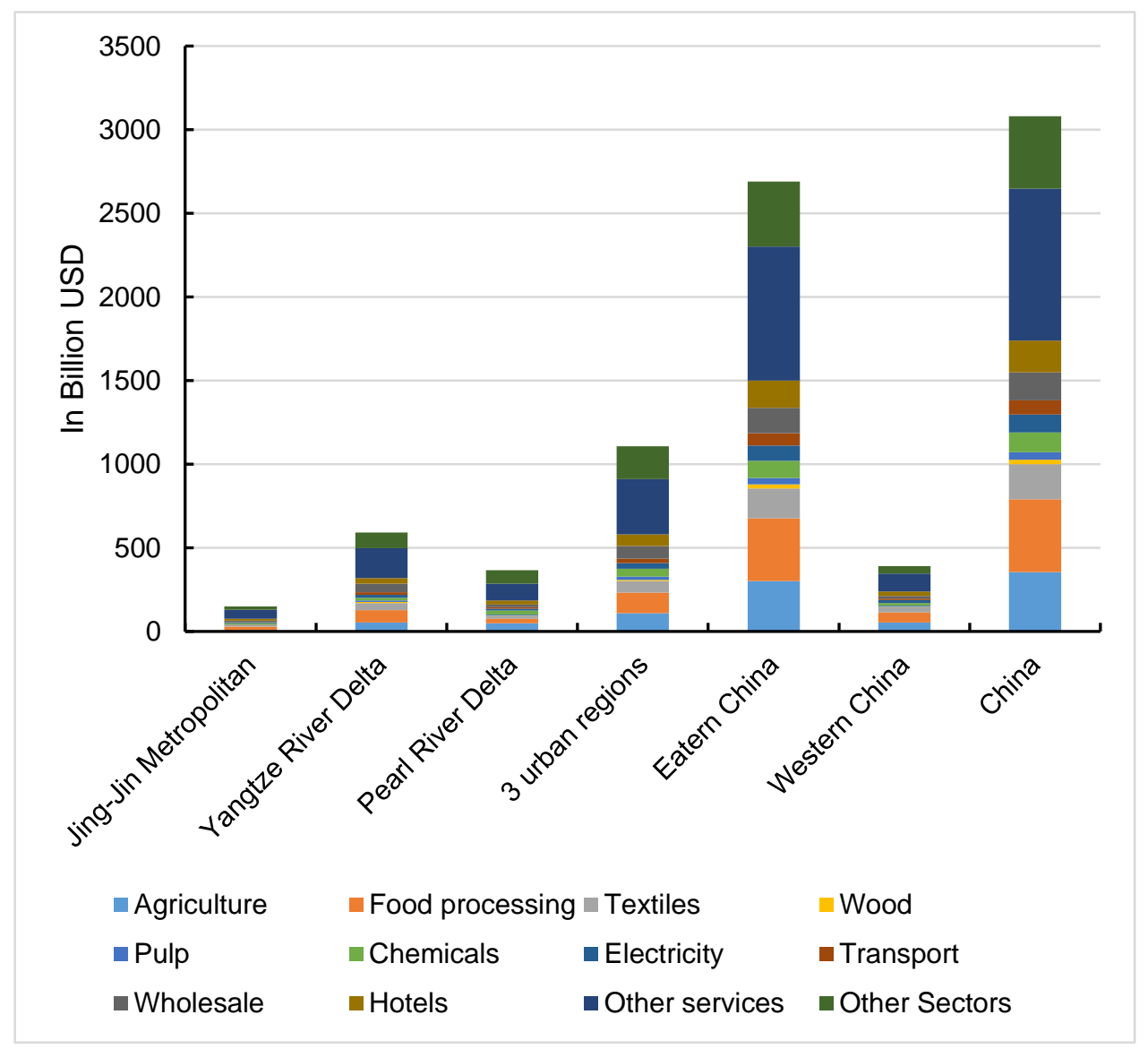

Supplementary Figure 6. Total final consumption expenditure by households of sub-national regions in China in 2010. Three major urban regions in China, i.e. Beijing-Tianjin metropolitan, Yangtze River Delta and Pearl River Delta, respectively take 38.6\% and 33.2\% total household consumption in Eastern China and whole mainland China in the year of 2010 from China MRIO table ${ }^{1}$. These numbers grew to $41.1 \%$ and $35.9 \%$ in 2012 according to data in 2012 Chinese regional input-output tables ${ }^{3}$. 


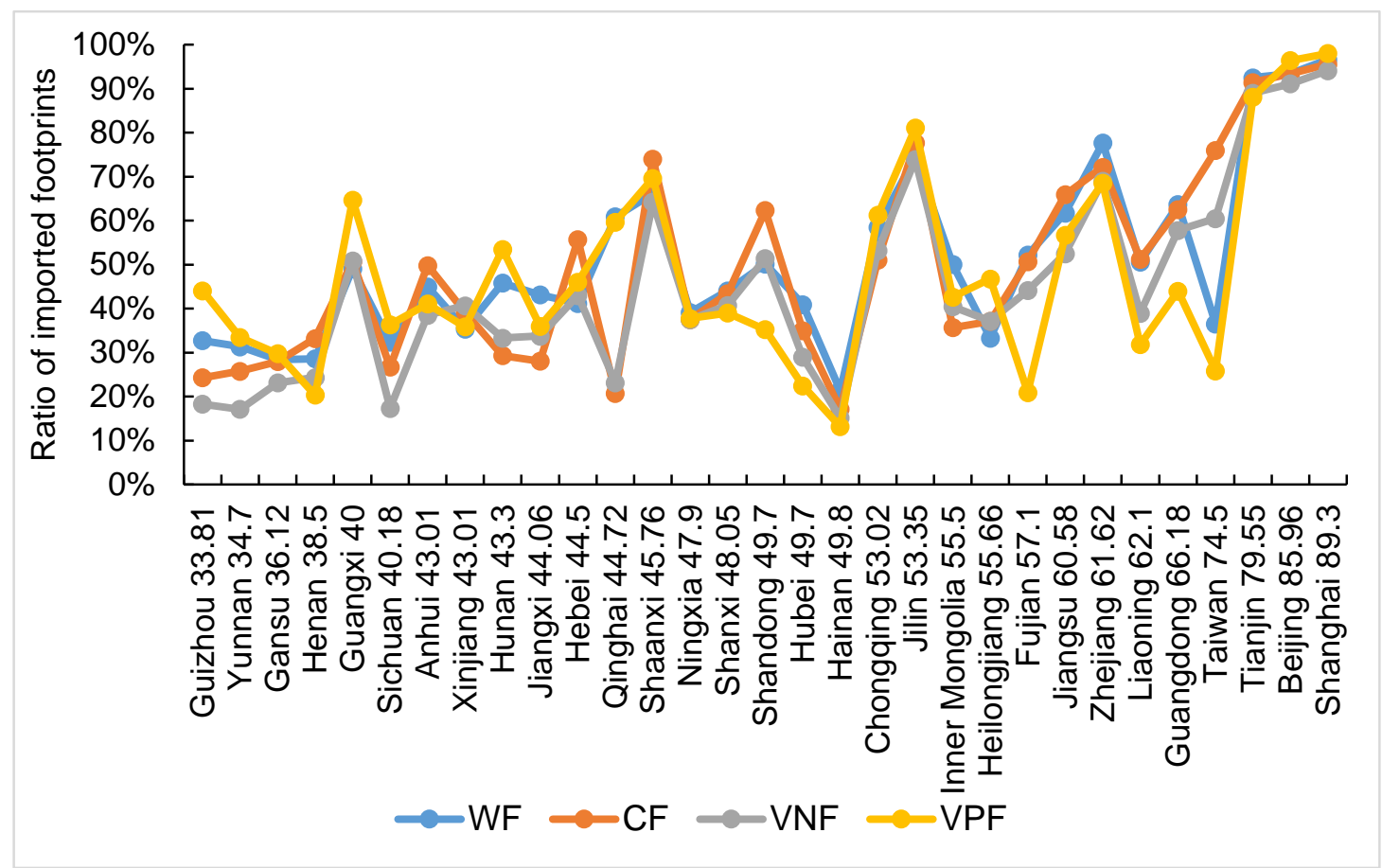

Supplementary Figure 7. Relationship of imported footprint ratios and urbanization. The imported footprint ratios are the proportions of imported footprints (that supplied from outside regions but consumed inside that region) in the total footprints in that region. 

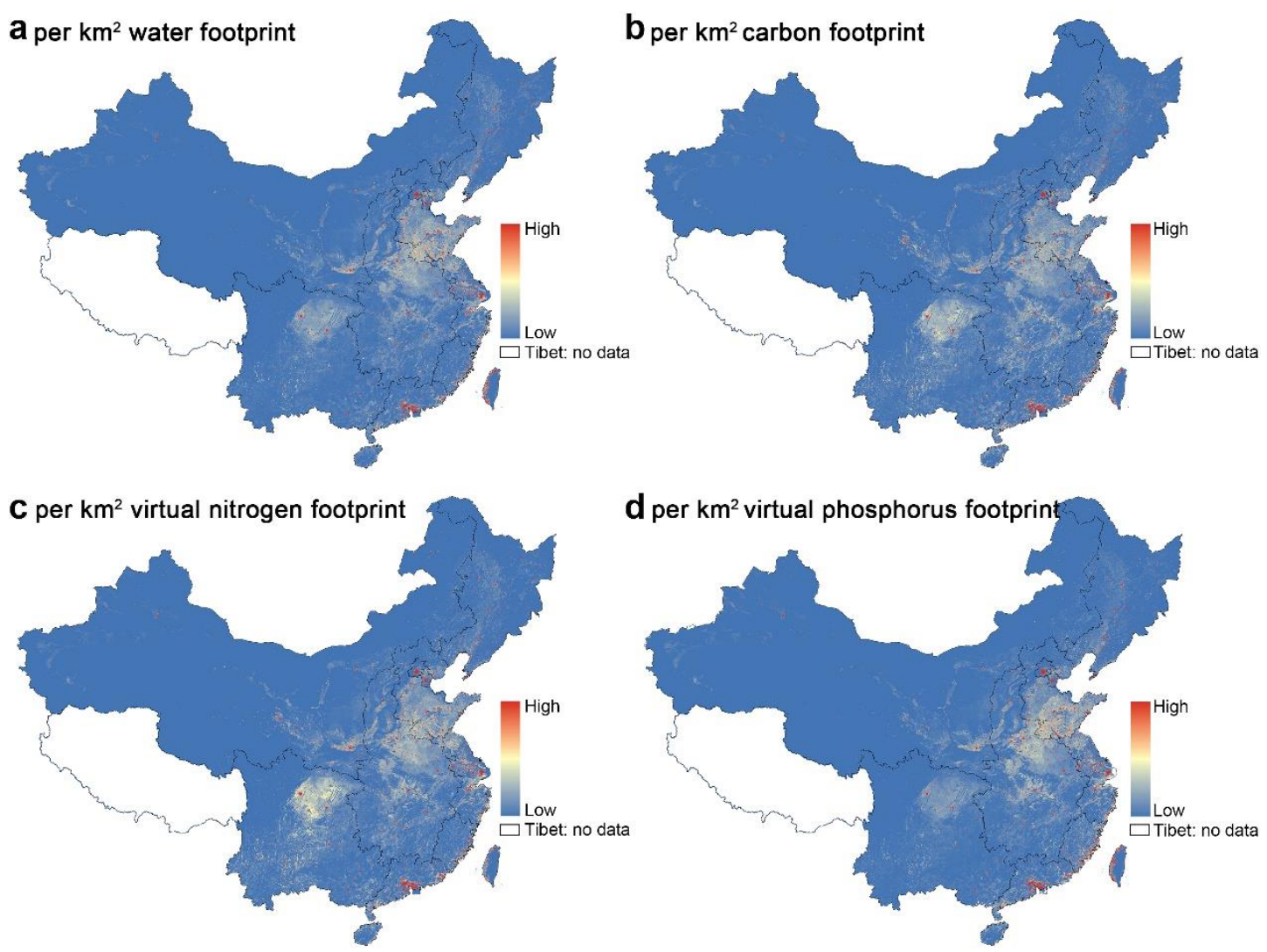

Supplementary Figure 8. The water(a), carbon(b), reactive nitrogen(c), and phosphorus footprint(c) in per unit area $\left(1 \mathbf{k m}^{2}\right)$. 


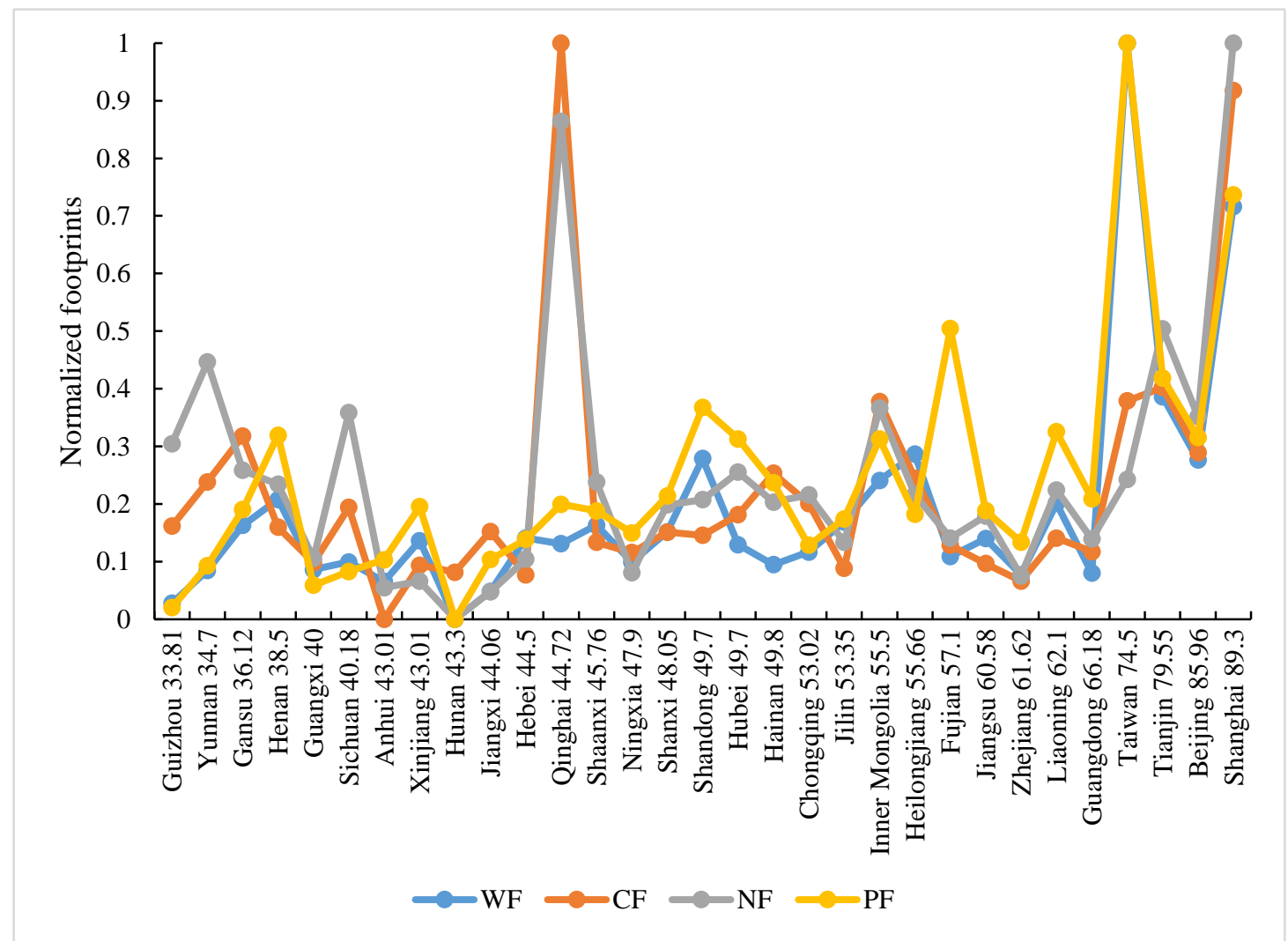

\section{Supplementary Figure 9. Relationship of standardized per capita footprints and} urbanization. Water footprint (WF), carbon footprint (CF), nitrogen footprint (NF) and phosphorus footprint (PF) were separately standardized by the min-max scaling method and thus ranging from zero to one. 


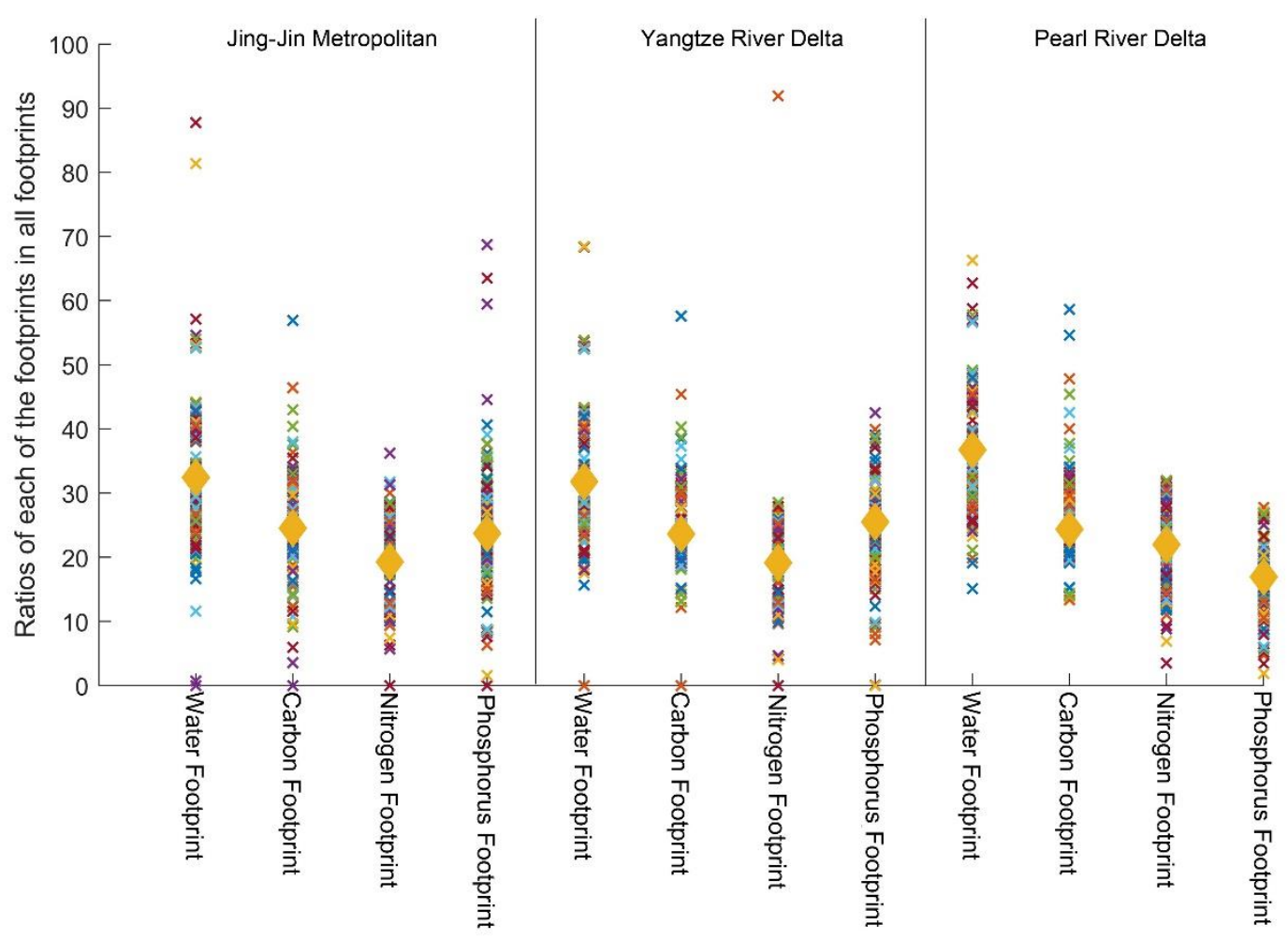

Supplementary Figure 10. The ratios each of the four footpirnts in the summary of normalized footprints. Crossed dots $(\times)$ show the regional and sectoral footprint of each urban region and the diamond signals $(\diamond)$ show the average levels. 

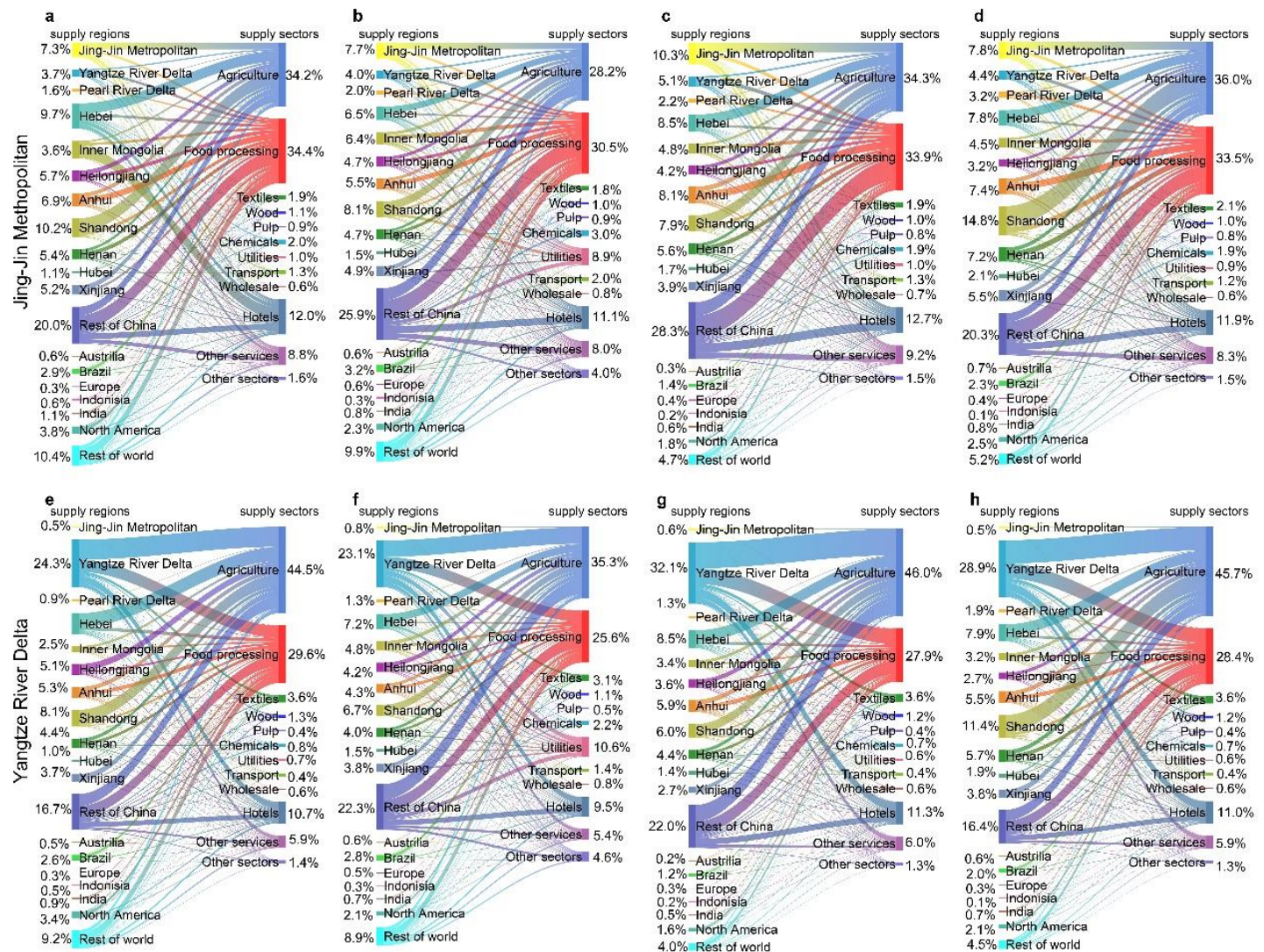

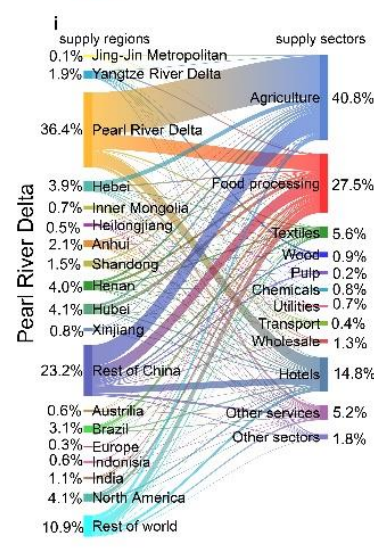

Water footprint

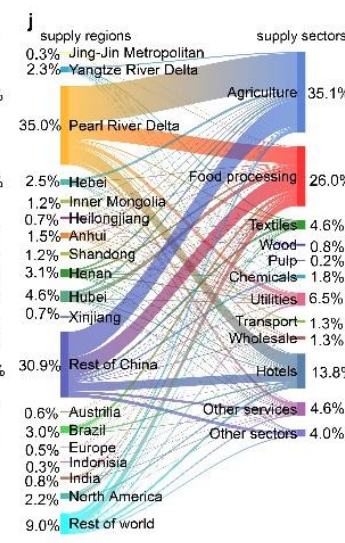

Carbon footprint

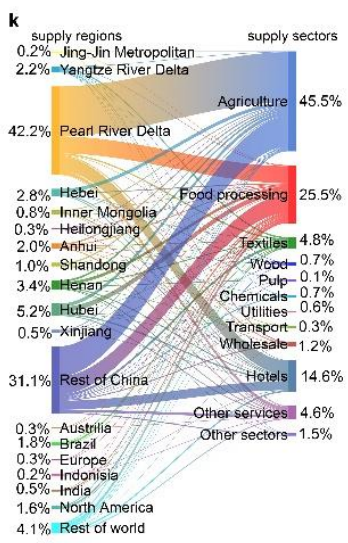

Nitrogen footprint

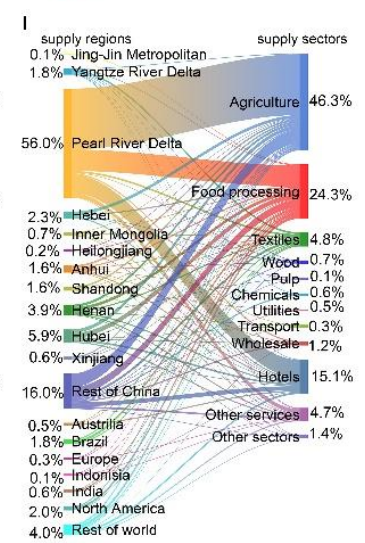

Phosphorus footprint

Supplementary Figure 11. Footprint supply chain of food-related household consumption by region/country and sector, for the three urban regions. The Sankey diagrams show the flow networks of water footprint (a, e, i), carbon footprint ( $b, f, j)$, reactive nitrogen footprint (c, g, k), and phosphorus footprint $(\mathrm{d}, \mathrm{h}, \mathrm{l})$ for Jing-Jin Metropolitan (a, b, c, d), Yangtze River Delta (e, f, g, h) and Pearl River Delta (i, j, k, l). Sectoral distribution of carbon footprints in three urban regions are more diverse than the other three footprints. More proportions of carbon footprints are allocated to utilities, transport, food processing and other sectors: but those allocated to the agriculture sector are about $5-10 \%$ less than other footprints. 


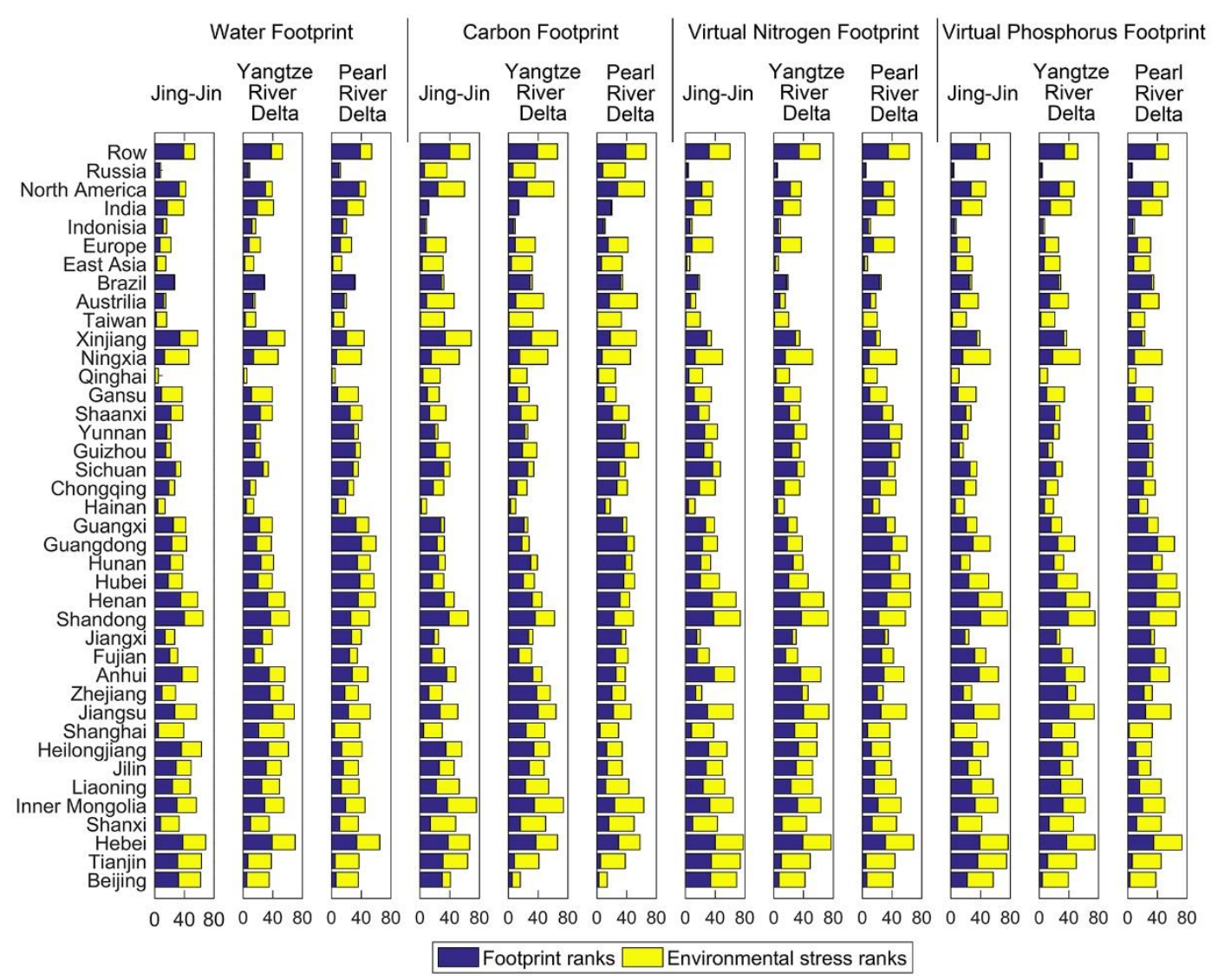

Supplementary Figure 12. Comparison of preferential mitigation regions of four footprints in three urban regions. The regional values of footprints and environmental stress are ranked within each of the urban regions, larger ranks denote higher priority and vice versa. Both the footprints and environmental stress are ranked among 40 regions from 1 to 40 : the ranks get larger as the regions have larger weight in the supply chain or the environmental risk get higher. 


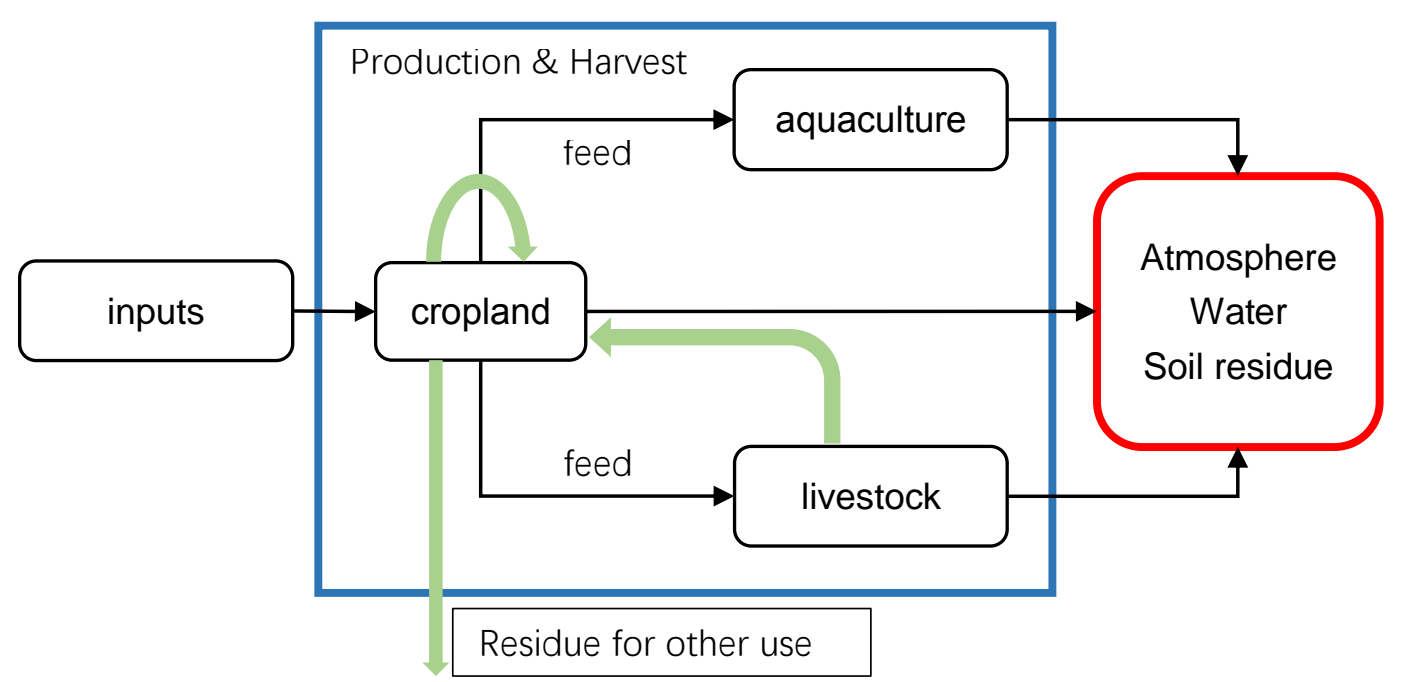

Supplementary Figure 13. Block diagram of material flows of agriculture sub-systems. The red block is boundary of environment, blue block is the boundary of agriculture activities including the production and harvest. 


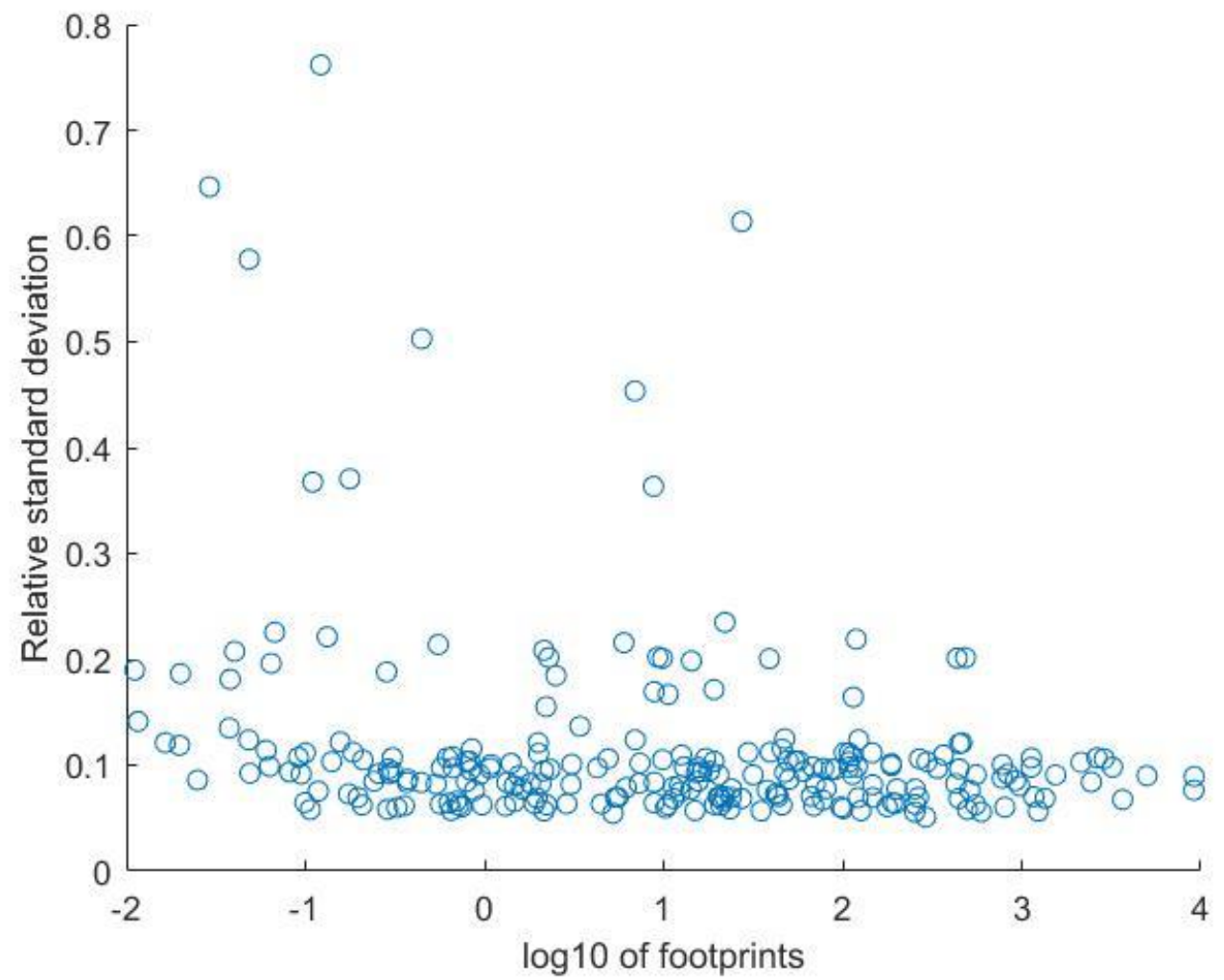

Supplementary Figure 14. Relative standard deviation (RSD) of footprints of food consumption in three urban regions. 


\section{Supplementary Tables}

Supplementary Table 1. The quantification of phosphorus $(P)$ and nitrogen $(N)$ emissions.

\begin{tabular}{|c|c|c|}
\hline \multicolumn{3}{|c|}{ Vegetable food production } \\
\hline Emission sources & Mathematical equations & Data description \\
\hline 1. From straw reuse & $\begin{array}{l}\mathrm{PN} 1=\mathrm{D} 1 \times(1 / \mathrm{P} 1-1) \times \mathrm{P} 2 \times \\
\mathrm{P} 3 \times \mathrm{P} 4 \times \mathrm{P} 5\end{array}$ & $\begin{array}{l}\text { D1: food production; } \\
\text { P1: economic efficient of plant; } \\
\text { P2: shoot part of plant; } \\
\text { P3: ratio of reuse of straw; } \\
\text { P4: P or N content of straw and root; } \\
\text { P5: gaseous emission, runoff and } \\
\text { leach rate of organic fertilizer of P or } \\
\text { N. }\end{array}$ \\
\hline 2. From manure reuse & $\begin{array}{l}\mathrm{PN} 2 \mathrm{a}=\mathrm{D} 2 \times \mathrm{P} 6 \times \mathrm{P} 7 \times \mathrm{P} 5 \\
\mathrm{PN} 2 \mathrm{~b}=\mathrm{D} 3 \times \mathrm{P} 8 \times \mathrm{P} 9 \times \mathrm{P} 5\end{array}$ & $\begin{array}{l}\text { D2: rural population; } \\
\text { D3: livestock and poultry on hand or } \\
\text { output; } \\
\text { P6: annual P or N content in excreta } \\
\text { of people; } \\
\text { P7: ratio of reuse of excreta of rural } \\
\text { population; } \\
\text { P8: annual P or N content in excreta } \\
\text { of animal; } \\
\text { P9: ratio of reuse of excreta of } \\
\text { animal; }\end{array}$ \\
\hline 3. From irrigation & $\begin{array}{l}\mathrm{PN} 3=\mathrm{D} 4 \times \mathrm{P} 10 \times \mathrm{P} 11 \times \mathrm{P} 12 \\
\times \mathrm{P} 5\end{array}$ & $\begin{array}{l}\text { D4: sowing area of plants; } \\
\text { P10: irrigation rate; } \\
\text { P11: P or N content in water; } \\
\text { P12: cost ratios of each type of food } \\
\text { in the total cost of irrigation. }\end{array}$ \\
\hline $\begin{array}{l}\text { 4. From chemical } \\
\text { fertilizer }\end{array}$ & $\begin{array}{l}\mathrm{PN} 4=\mathrm{D} 4 \times \mathrm{P} 13 \times \mathrm{P} 14 \\
\text { Where D4 } \\
\mathrm{P} 13=\mathrm{D} 5+\mathrm{D} 6 / 3\end{array}$ & $\begin{array}{l}\text { D5: P or } \mathrm{N} \text { fertilizer; } \\
\text { D6: compound fertilizer; } \\
\text { P13: chemical fertilizer application } \\
\text { rate; } \\
\text { P14: gaseous emission, runoff and } \\
\text { leach rate of chemical fertilizer. }\end{array}$ \\
\hline 5. From deposition & $\mathrm{PN} 5=\mathrm{D} 4 \times \mathrm{P} 15 \times \mathrm{P} 14$ & $\begin{array}{l}\text { P15: atmospheric } \mathrm{P} \text { or } \mathrm{N} \text { deposition } \\
\text { per unit of land. }\end{array}$ \\
\hline $\begin{array}{l}\text { 6. From nitrogen } \\
\text { fixation }\end{array}$ & $\mathrm{N} 6=\mathrm{D} 4 \times \mathrm{P} 16 \times \mathrm{P} 14$ & P16: $\mathrm{N}$ fixation rate of pulse. \\
\hline $\begin{array}{l}\text { 7. From pesticide } \\
\text { application }\end{array}$ & $\mathrm{P} 7=\mathrm{D} 4 \times \mathrm{P} 17 \times \mathrm{P} 18 \times \mathrm{P} 14$ & $\begin{array}{l}\text { P17: pesticide application rate; } \\
\text { P18: P content in pesticide. }\end{array}$ \\
\hline 8. From seed & $\mathrm{PN} 8=\mathrm{D} 0 \times \mathrm{D} 1 \times \mathrm{P} 0 \times \mathrm{P} 5$ & $\begin{array}{l}\text { D0: sown amount per unit } \\
\text { production; }\end{array}$ \\
\hline
\end{tabular}




\begin{tabular}{|c|c|c|}
\hline & & P0: $\mathrm{N}$ or $\mathrm{P}$ content in seed. \\
\hline 9. From energy use & $\mathrm{N} 9=\mathrm{D} 7 \times \mathrm{P} 19$ & $\begin{array}{l}\text { D7: energy use in vegetable food } \\
\text { production; } \\
\text { P19: emission factors of energy use. }\end{array}$ \\
\hline \multicolumn{3}{|c|}{ Animal food production } \\
\hline Emission sources & Mathematical equations & Data description \\
\hline $\begin{array}{l}\text { 10. From manure } \\
\text { management }\end{array}$ & $\begin{array}{l}\text { PN10=D3 } \times \text { P8-PN2b- } \\
\text { PN9; } \\
\text { Where PN9 is for other } \\
\text { usage or emission }\end{array}$ & \\
\hline 11. From energy use & $\mathrm{N} 11=\mathrm{D} 8 \times \mathrm{P} 19$ & $\begin{array}{l}\text { D8: energy use in animal food } \\
\text { production. }\end{array}$ \\
\hline \multicolumn{3}{|c|}{ Aquatic food production } \\
\hline Emission sources & Mathematical equations & Data description \\
\hline 12. From feed loss & $\begin{array}{l}\mathrm{PN} 12=\mathrm{D} 9 \times \mathrm{P} 20 \times \mathrm{P} 21 \times \\
\mathrm{P} 22 \times \mathrm{P} 23\end{array}$ & $\begin{array}{l}\text { D9: aquaculture production; } \\
\text { P20: feed conversion ratio; } \\
\text { P21: P or N content in feed; } \\
\text { P22: feed loss rate; } \\
\text { P23: gaseous emission, runoff or } \\
\text { leach rate of wasted feed. }\end{array}$ \\
\hline 13. From energy use & $\mathrm{N} 13=\mathrm{D} 10 \times \mathrm{P} 19$ & $\begin{array}{l}\text { D10: energy use in aquatic food } \\
\text { production. }\end{array}$ \\
\hline \multicolumn{3}{|l|}{ Industrial production } \\
\hline $\begin{array}{l}\text { 14. From N- or P- } \\
\text { containing products }\end{array}$ & $\mathrm{NP} 14=\mathrm{D} 11 \times \mathrm{P} 24$ & $\begin{array}{l}\text { D11: production amount of industrial } \\
\text { products; } \\
\text { P24: gaseous emission, runoff or } \\
\text { leach rate of industrial products. }\end{array}$ \\
\hline 15. From energy use & $\mathrm{N} 15=\mathrm{D} 12 \times \mathrm{P} 19$ & $\begin{array}{l}\text { D12: energy use in industrial } \\
\text { production. }\end{array}$ \\
\hline \multicolumn{3}{|c|}{ Waste water and solid waste treatment } \\
\hline $\begin{array}{l}\text { 16. From treatment } \\
\text { plant }\end{array}$ & $\mathrm{NP} 16=\mathrm{D} 13 \times \mathrm{P} 25 \times \mathrm{P} 26$ & $\begin{array}{l}\text { D13: waste water or solid waste } \\
\text { amount; } \\
\text { P25: discharge rate of } \mathrm{N} \text { or P; } \\
\text { P26: gaseous emission, runoff or } \\
\text { leach rate of waste discharge. }\end{array}$ \\
\hline 17. From energy use & $\mathrm{N} 17=\mathrm{D} 14 \times \mathrm{P} 19$ & D14: energy use in waste treatment. \\
\hline \multicolumn{3}{|l|}{ Forest and grassland } \\
\hline 18. From deposition & $\mathrm{NP} 18=\mathrm{D} 15 \times \mathrm{P} 15 \times \mathrm{P} 14$ & D15: area of forest and grassland. \\
\hline 19. From $\mathrm{N}$ fixation & $\mathrm{N} 19=\mathrm{D} 15 \times \mathrm{P} 27 \times \mathrm{P} 28$ & $\begin{array}{l}\text { P27: } \mathrm{N} \text { fixation rate of forest and } \\
\text { grassland; } \\
\text { P28: gaseous emission, runoff or } \\
\text { leach rate of forest and grassland. }\end{array}$ \\
\hline
\end{tabular}

Note:

1. D1-D5 are activity data that can be collected from the statistics; 
2. P1-P22 are parameters mainly collected by the following previous research:

Cui, S., Shi, Y., Groffman, P. M., Schlesinger, W. H. \& Zhu, Y.-G. Centennial-scale analysis of the creation and fate of reactive nitrogen in China (1910-2010). Proceedings of the National Academy of Sciences 110, 2052-2057 (2013).

Gu, B., Ju, X., Chang, J., Ge, Y. \& Vitousek, P. M. Integrated reactive nitrogen budgets and future trends in China. Proceedings of the National Academy of Sciences 112, 87928797 (2015).

$\mathrm{Hu}$, Y. et al. Evaluating agricultural grey water footprint with modeled nitrogen emission data. Resources, Conservation and Recycling 138, 64-73, (2018).

Liu, X. et al. Intensification of phosphorus cycling in China since the 1600s. Proceedings of the National Academy of Sciences 113, 2609-2614 (2016).

Cui, S. et al. Changing urban phosphorus metabolism: evidence from Longyan City, China.

Science of the Total Environment 536, 924-932 (2015).

3 . The emissions are assumed to have a 10-30\% coefficient of variation according to previous study (Cui, S., Shi, Y., Groffman, P. M. et al, 2013).

4. Gaseous emission include $\mathrm{NH}_{3}, \mathrm{~N}_{2} \mathrm{O}$ and $\mathrm{NOx}$ and is only applied to nitrogen biogeochemical cycle, due to energy combustion, volatilization, nitrification or denitrification processes.

5. Pet rearing and deposition to coastal regions are not belong to economic sectors, and thus didn't included in this research. 
Supplementary Table 2. Sources of environmental accounts by sector and by region.

\begin{tabular}{ll|llll}
\hline Sectors & Regions & Water & Carbon & Reactive nitrogen & Phosphorus \\
\hline \multirow{2}{*}{ Cropland $^{\mathrm{a}}$} & Mainland China & This research & This research & This research & This research \\
& Other regions & WIOD & WIOD & WIOD $^{\mathrm{d}}$ & This research \\
Aquaculture $^{\mathrm{b}}$ & Mainland China & - & This research & - & This research \\
& Other regions & - & WIOD & - & This research \\
Livestock $^{\mathrm{c}}$ & Mainland China & This research & This research & This research & This research \\
& Other regions & WIOD & WIOD & WIOD $^{\mathrm{d}}$ & This research \\
\multirow{2}{*}{ Other sectors } & Mainland China & This research & This research & This research $^{*}$ & This research \\
& Other regions & WIOD & WIOD & WIOD $^{\mathrm{d}}$ & This research \\
\hline
\end{tabular}

\section{Notes:}

${ }^{a}$ Nine categories of products include rice (three types), wheat (one type), other cereals (five types), starchy roots (two types), sugar crops (two types), pulses (four types), oil crops (12 types), vegetables (21 types) and fruits (18 types).

${ }^{\mathrm{b}}$ Five categories of products include fish, shrimp and crab, shell, seaweed and others: each of them include freshwater and seawater cultivation.

${ }^{\mathrm{c}}$ Seven categories of products include bovine meat, milk, mutton and goat meat, pork, poultry meat and eggs.

${ }^{\mathrm{d}}$ Atmospheric emissions come from WIOD database, while emissions to waterbody are accounted in this study.

Supplementary Table 3. The definitions of the aggregated regions appeared in the text.

\begin{tabular}{ll}
\hline Aggregated regions & Description \\
\hline Jing-Jin Metropolitan & Beijing, Tianjin \\
Yangtze River Delta & Shanghai, Jiangsu, Zhejiang \\
Pearl River Delta & Guangdong \\
Outside China & All other countries or region except mainland \\
& China and Taiwan province of China \\
Rest of world (Row, presented in Figure 2 \& 3) & All other regions except the 40 regions in \\
& WIOD, as defined by WIOD \\
East Asia & Japan, Korea \\
North America & United States, Canada, Mexico \\
Europe & 27 countries in European Union and United \\
& Kingdom \\
Other regions (presented in Figure 4) & All other regions except those listed before \\
\hline
\end{tabular}




\section{Supplementary Table 4. Prioritized regions for the three Chinese urban regions to reduce four}

food-related footprints. The cells are highlighted in blue, green, orange and red to correspond with this region being crucial for none, one, two and three urban regions, respectively.

\begin{tabular}{|c|c|c|c|c|}
\hline & Water Footprint & Carbon Footprint & Nitrogen Footprint & $\begin{array}{l}\text { Phosphorus } \\
\text { Footprint }\end{array}$ \\
\hline Hebei & $\begin{array}{l}\text { Beijing-Tianjin, } \\
\text { Yangtze River } \\
\text { Delta, Pearl River } \\
\text { Delta }\end{array}$ & $\begin{array}{l}\text { Beijing-Tianjin, } \\
\text { Yangtze River } \\
\text { Delta, Pearl River } \\
\text { Delta }\end{array}$ & $\begin{array}{l}\text { Beijing-Tianjin, } \\
\text { Yangtze River } \\
\text { Delta, Pearl River } \\
\text { Delta }\end{array}$ & $\begin{array}{l}\text { Beijing-Tianjin, } \\
\text { Yangtze River } \\
\text { Delta, Pearl River } \\
\text { Delta }\end{array}$ \\
\hline $\begin{array}{l}\text { Inner } \\
\text { Mongolia }\end{array}$ & & $\begin{array}{l}\text { Beijing-Tianjin, } \\
\text { Yangtze River } \\
\text { Delta, Pearl River } \\
\text { Delta }\end{array}$ & $\begin{array}{l}\text { Beijing-Tianjin, } \\
\text { Yangtze River } \\
\text { Delta }\end{array}$ & $\begin{array}{l}\text { Beijing-Tianjin, } \\
\text { Yangtze River } \\
\text { Delta }\end{array}$ \\
\hline Heilongjiang & $\begin{array}{l}\text { Beijing-Tianjin, } \\
\text { Yangtze River } \\
\text { Delta }\end{array}$ & Beijing-Tianjin & & \\
\hline Anhui & Beijing-Tianjin & & $\begin{array}{l}\text { Beijing-Tianjin, } \\
\text { Yangtze River } \\
\text { Delta }\end{array}$ & Beijing-Tianjin \\
\hline Jiangsu & $\begin{array}{l}\text { Yangtze River } \\
\text { Delta, Pearl River } \\
\text { Delta }\end{array}$ & $\begin{array}{l}\text { Yangtze River } \\
\text { Delta }\end{array}$ & $\begin{array}{l}\text { Beijing-Tianjin, } \\
\text { Yangtze River } \\
\text { Delta, Pearl River } \\
\text { Delta }\end{array}$ & $\begin{array}{l}\text { Beijing-Tianjin, } \\
\text { Yangtze River } \\
\text { Delta }\end{array}$ \\
\hline Shandong & $\begin{array}{l}\text { Beijing-Tianjin, } \\
\text { Yangtze River } \\
\text { Delta }\end{array}$ & $\begin{array}{l}\text { Beijing-Tianjin, } \\
\text { Yangtze River } \\
\text { Delta }\end{array}$ & $\begin{array}{l}\text { Beijing-Tianjin, } \\
\text { Yangtze River } \\
\text { Delta }\end{array}$ & $\begin{array}{l}\text { Beijing-Tianjin, } \\
\text { Yangtze River } \\
\text { Delta, Pearl River } \\
\text { Delta }\end{array}$ \\
\hline Henan & $\begin{array}{l}\text { Beijing-Tianjin, } \\
\text { Yangtze River } \\
\text { Delta, Pearl River } \\
\text { Delta }\end{array}$ & & $\begin{array}{l}\text { Beijing-Tianjin, } \\
\text { Yangtze River } \\
\text { Delta, Pearl River } \\
\text { Delta }\end{array}$ & $\begin{array}{l}\text { Beijing-Tianjin, } \\
\text { Yangtze River } \\
\text { Delta, Pearl River } \\
\text { Delta }\end{array}$ \\
\hline Hubei & Pearl River Delta & Pearl River Delta & Pearl River Delta & Pearl River Delta \\
\hline Xinjiang & $\begin{array}{l}\text { Beijing-Tianjin, } \\
\text { Yangtze River } \\
\text { Delta }\end{array}$ & $\begin{array}{l}\text { Beijing-Tianjin, } \\
\text { Yangtze River } \\
\text { Delta, Pearl River } \\
\text { Delta }\end{array}$ & & \\
\hline Row & & $\begin{array}{l}\text { Beijing-Tianjin, } \\
\text { Yangtze River } \\
\text { Delta, Pearl River } \\
\text { Delta }\end{array}$ & $\begin{array}{l}\text { Beijing-Tianjin, } \\
\text { Yangtze River } \\
\text { Delta, Pearl River } \\
\text { Delta }\end{array}$ & \\
\hline
\end{tabular}


Supplementary Table 5. Environmental effect on WF, CF, VNF, VPF of different strategies

\begin{tabular}{lcccc}
\hline & Jing-Jin Metropolitan & Yangtze River Delta & Pearl River Delta & \\
\hline Baseline & & & & Units \\
\hline WF & 32.8 & 119.9 & 54.1 & $\mathrm{G} \mathrm{m}^{3}$ \\
CF & 27.2 & 102.7 & 55.6 & $\mathrm{Tg}$ \\
VNF & 0.5 & 1.8 & 0.9 & $\mathrm{Tg}$ \\
VPF & 0.1 & 0.5 & 0.3 & $\mathrm{Tg}$ \\
\hline Reduce half of the food loss and waste & & & $\mathrm{Units}$ \\
\hline WF & 31.6 & 114.4 & 51.8 & $\mathrm{G} \mathrm{m}$ \\
CF & 26.1 & 97.2 & 52.8 & $\mathrm{Tg}$ \\
VNF & 1.7 & 0.9 & $\mathrm{Tg}$ \\
VPF & 0.5 & 0.5 & 0.3 & $\mathrm{Tg}$ \\
\hline Optimize fertilizer application in mainland China & & & $\mathrm{Units}$ \\
\hline WF & 31.2 & 114.1 & 51.7 & $\mathrm{G} \mathrm{m}{ }^{3}$ \\
CF & 98.1 & 53.4 & $\mathrm{Tg}$ \\
VNF & 25.9 & 1.4 & 0.7 & $\mathrm{Tg}$ \\
VPF & 0.4 & 0.4 & 0.2 & $\mathrm{Tg}$ \\
\hline Combination of the two strategies & & & $\mathrm{Units}$ \\
\hline WF & 30.0 & 108.6 & 49.4 & $\mathrm{G} \mathrm{m}{ }^{3}$ \\
CF & 24.8 & 92.8 & 50.7 & $\mathrm{Tg}$ \\
VNF & 0.4 & 1.3 & 0.7 & $\mathrm{Tg}$ \\
VPF & 0.1 & 0.4 & 0.2 & $\mathrm{Tg}$ \\
\hline
\end{tabular}

Notes: Strategy 1 means a mediate (half) reduction of food loss and waste, so the ratio of food loss and waste reduce from $20.7 \%$ to $10.35 \%$. Strategy 2 means a mediate reduction ( $50 \%$ of the potential) of avoidable fertilizer application, and a mediate increase ( $50 \%$ of the potential) of reuse of straw and manure as the replacement of chemical fertilizer. 
Supplementary Table 6. Regions divided by Hu Huanyong line and the areas assigned to east and west China.

\begin{tabular}{llllll}
\hline & \multicolumn{3}{c}{ Area $(\mathrm{k}$ ha) } & \multicolumn{2}{c}{ Ratio } \\
Regions & East & West & Total & East & West \\
\hline Beijing & 16.2 & 0.2 & 16.4 & 0.99 & 0.01 \\
Hebei & 136.8 & 49.2 & 186.0 & 0.74 & 0.26 \\
Heilongjiang & 365.6 & 94.7 & 460.3 & 0.79 & 0.21 \\
Jilin & 193.3 & 0.3 & 193.6 & 1.00 & 0.00 \\
Inner & 107.3 & 1051.6 & 1158.9 & 0.09 & 0.91 \\
Mongolia & & & & & \\
Shanxi & 68.8 & 88.3 & 157.0 & 0.44 & 0.56 \\
Shaanxi & 67.3 & 141.3 & 208.7 & 0.32 & 0.68 \\
Sichuan & 161.4 & 345.7 & 507.1 & 0.32 & 0.68 \\
Yunnan & 337.9 & 69.4 & 407.4 & 0.83 & 0.17 \\
\hline
\end{tabular}

Supplementary Table 7. Inventory comparing this with other studies for Mainland China

\begin{tabular}{llllll}
\hline & & Water & Carbon & Reactive nitrogen & Phosphorus \\
\hline \multirow{4}{*}{ This study } & Amount & $1320 \mathrm{Gt}$ & $\begin{array}{l}\text { Total } \mathrm{CO}_{2} \mathrm{e} 8984 \mathrm{Tg} \\
\text { Energy } \mathrm{CO}_{2}: 7845 \mathrm{Tg}\end{array}$ & $33 \mathrm{Tg}$ & $7 \mathrm{Tg}$ \\
& Year & 2010 & 2010 & 2010 & 2010 \\
\cline { 2 - 6 } Other studies & Amount & $1207 \mathrm{Gt}^{4}$ & $7334 \mathrm{Tg}^{5}$ & $32 \mathrm{Tg}^{7} ;$ & $7 \mathrm{Tg}^{9}$ \\
& & & $7724 \mathrm{Tg}^{6}$ & $41 \mathrm{Tg}^{8}$ & 2012 \\
\hline
\end{tabular}


Supplementary Table 8. The indicators of environmental and resource stress.

\begin{tabular}{|c|c|c|c|c|}
\hline Stresses & Water resource & Climate mitigation & Surface water $\mathrm{Nr}$ & Surface w. P \\
\hline Indicators & $\begin{array}{c}\text { Water scarcity } \\
\text { index, } 1\end{array}$ & $\begin{array}{l}\text { Per capita GHG } \\
\text { emission, t/cap }\end{array}$ & $\begin{array}{c}\text { Water Nr load, } \\
\mathrm{mg} / \mathrm{L}\end{array}$ & $\begin{array}{c}\text { Water P } \\
\text { load, mg/L }\end{array}$ \\
\hline Beijing & 1.41 & 4.91 & 21.73 & 7.43 \\
\hline Tianjin & 2.41 & 11.62 & 57.94 & 25.07 \\
\hline Hebei & 1.82 & 9.01 & 52.16 & 16.55 \\
\hline Shanxi & 0.58 & 14.94 & 14.27 & 4.12 \\
\hline Inner Mongolia & 0.59 & 22.12 & 9.09 & 2.54 \\
\hline Liaoning & 0.57 & 10.44 & 6.37 & 2.52 \\
\hline Jilin & 0.31 & 7.16 & 2.84 & 0.86 \\
\hline Heilongjiang & 0.60 & 7.50 & 3.46 & 1.00 \\
\hline Shanghai & 3.79 & 8.84 & 8.12 & 2.69 \\
\hline Jiangsu & 1.13 & 8.42 & 16.88 & 5.83 \\
\hline Zhejiang & 0.24 & 6.76 & 1.34 & 0.50 \\
\hline Anhui & 0.32 & 5.47 & 4.16 & 1.33 \\
\hline Fujian & 0.18 & 6.56 & 1.89 & 0.80 \\
\hline Jiangxi & 0.20 & 3.96 & 0.90 & 0.40 \\
\hline Shandong & 0.58 & 8.84 & 33.34 & 13.05 \\
\hline Henan & 0.43 & 5.56 & 11.30 & 3.87 \\
\hline Hubei & 0.25 & 5.65 & 3.68 & 1.37 \\
\hline Hunan & 0.23 & 4.30 & 1.69 & 0.59 \\
\hline Guangdong & 0.31 & 4.87 & 2.70 & 1.11 \\
\hline Guangxi & 0.23 & 3.74 & 1.68 & 0.65 \\
\hline Hainan & 0.16 & 4.19 & 1.46 & 0.55 \\
\hline Chongqing & 0.12 & 5.57 & 2.77 & 0.83 \\
\hline Sichuan & 0.09 & 4.21 & 1.47 & 0.48 \\
\hline Guizhou & 0.09 & 7.01 & 1.58 & 0.41 \\
\hline Yunnan & 0.07 & 3.53 & 1.91 & 0.47 \\
\hline Shaanxi & 0.22 & 7.96 & 1.74 & 0.43 \\
\hline Gansu & 0.69 & 6.16 & 3.27 & 1.12 \\
\hline Qinghai & 0.05 & 8.06 & 2.29 & 0.49 \\
\hline Ningxia & 3.11 & 21.71 & 49.25 & 15.83 \\
\hline Xinjiang & 0.57 & 15.00 & 1.01 & 0.32 \\
\hline Taiwan & 0.21 & 11.58 & 2.67 & 0.93 \\
\hline Australia & 0.03 & 15.83 & 1.13 & 1.32 \\
\hline Brazil & 0.01 & 2.17 & 0.29 & 0.22 \\
\hline East Asia & 0.19 & 8.99 & 0.61 & 1.10 \\
\hline Europe & 0.21 & 8.93 & 5.35 & 0.89 \\
\hline Indonesia & 0.06 & 1.72 & 0.59 & 0.20 \\
\hline India & 0.34 & 1.58 & 3.40 & 1.95 \\
\hline North America & 0.15 & 15.53 & 1.76 & 0.96 \\
\hline Russia & 0.01 & 10.19 & 0.19 & 0.07 \\
\hline Row & 0.21 & 8.93 & 5.35 & 0.89 \\
\hline
\end{tabular}


Supplementary Table 9. Postharvest food loss rate of different stages of China in 2010

\begin{tabular}{lllllll}
\hline & handling & storage & processing & distribution & consuming & Total \\
\hline Grain & $5 \%$ & $7.2 \%$ & $2.7 \%$ & $1.2 \%$ & $7.3 \%$ & $23.2 \%$ \\
Meat & $2.7 \%$ & $3.1 \%$ & $1.1 \%$ & $3 \%$ & $7.3 \%$ & $17.2 \%$ \\
Veg. \& fruit & & $15 \%$ & & $10 \%$ & $7.3 \%$ & $32.3 \%$ \\
\hline
\end{tabular}

Note: see refs. ${ }^{18}$ and ${ }^{19}$.

Supplementary Table 10. Per capita annual food expenditure and purchases of major commodities of urban household of high income households of China in 2010

\begin{tabular}{lllll}
\hline & Grain & Meat & Eggs & Vegetables \& fruit \\
\hline Expenditure $(¥)$ & 453.87 & 1142.37 & 117.74 & $289.58^{\mathrm{a}}$ \\
Purchases $(\mathrm{kg})$ & 81.53 & 49.72 & 10.00 & 170.34 \\
\hline
\end{tabular}

Note: ${ }^{\text {a }}$ Yearbook didn't provide this value and we estimated it with price and purchases.

Supplementary Table 11. Largest reduction rate of fertilizer uses without sacrificing crop yield

\begin{tabular}{lll}
\hline & fertilizer N & fertilizer P \\
\hline \multirow{3}{*}{ Wheat } & $20 \%^{20}$ in northwest, & $10 \sim 30 \%{ }^{22}$, \\
& $37.5 \%^{21}$ in central, & $20 \%{ }^{23}$ \\
\multirow{2}{*}{ Maize } & $60 \%^{21}$ in north & $10 \sim 30 \%{ }^{22}$, \\
& $70 \%^{20}$ in northwest, & $20 \%^{23}$ \\
Rice & $41.2^{21}$ in north & $10 \sim 30 \%$ \\
& $33 \%^{21}$ in central & $20 \%{ }^{23}$ \\
\hline
\end{tabular}


Supplementary Table 12. Current and potential of nutrient resources of residue and increment returning in different regions in China (\%)

\begin{tabular}{|c|c|c|c|c|c|c|}
\hline \multirow[t]{2}{*}{ Region } & \multicolumn{3}{|c|}{ Residue and straw } & \multicolumn{2}{|c|}{ manure } & \multirow[b]{2}{*}{ Increase by ${ }^{2}$} \\
\hline & $\mathrm{N}^{24}$ & $\mathrm{P}_{2} \mathrm{O}_{5}{ }^{24}$ & Increase by ${ }^{24}$ & $\mathrm{~N}^{25}$ & $\mathrm{P}_{2} \mathrm{O}_{5}{ }^{26,27}$ & \\
\hline Beijing & 47.3 & 47.3 & 55.9 & 50 & 45.8 & 22 \\
\hline Tianjin & 47.3 & 47.3 & 55.6 & 50 & 45.8 & 22 \\
\hline Hebei & 47.3 & 47.3 & 55.3 & 50 & 45.8 & 22 \\
\hline Shanxi & 55.7 & 55.7 & 39.4 & 50 & 45.8 & 22 \\
\hline Inner Mongolia & 15 & 15 & 282.8 & 50 & 45.8 & 22 \\
\hline Liaoning & 31.1 & 31.1 & 110.6 & 50 & 45.8 & 22 \\
\hline Jilin & 15 & 15 & 283.3 & 50 & 45.8 & 22 \\
\hline Heilongjiang & 35.1 & 35.1 & 92.3 & 50 & 45.8 & 22 \\
\hline Shanghai & 47.8 & 47.8 & 54.5 & 50 & 45.8 & 22 \\
\hline Jiangsu & 31.9 & 31.9 & 106.7 & 50 & 45.8 & 22 \\
\hline Zhejiang & 23.9 & 23.9 & 159.7 & 50 & 45.8 & 22 \\
\hline Anhui & 30.3 & 30.3 & 115.0 & 50 & 45.8 & 22 \\
\hline Fujian & 35.5 & 35.5 & 91.2 & 50 & 45.8 & 22 \\
\hline Jiangxi & 65.1 & 65.1 & 26.7 & 50 & 45.8 & 22 \\
\hline Shandong & 23.6 & 23.6 & 162.3 & 50 & 45.8 & 22 \\
\hline Henan & 34.6 & 34.6 & 94.9 & 50 & 45.8 & 22 \\
\hline Hubei & 38 & 38 & 81.8 & 50 & 45.8 & 22 \\
\hline Hunan & 20 & 20 & 200.0 & 50 & 45.8 & 22 \\
\hline Guangdong & 41.4 & 41.4 & 70.7 & 50 & 45.8 & 22 \\
\hline Guangxi & 15 & 15 & 282.3 & 50 & 45.8 & 22 \\
\hline Hainan & 38 & 38 & 82.1 & 50 & 45.8 & 22 \\
\hline Chongqing & 15 & 15 & 282.5 & 50 & 45.8 & 22 \\
\hline Sichuan & 14.3 & 14.3 & 299.4 & 50 & 45.8 & 22 \\
\hline Guizhou & 15 & 15 & 284.4 & 50 & 45.8 & 22 \\
\hline Yunnan & 15 & 15 & 281.7 & 50 & 45.8 & 22 \\
\hline Shannxi & 32 & 32 & 105.7 & 50 & 45.8 & 22 \\
\hline Gansu & 26.8 & 26.8 & 136.8 & 50 & 45.8 & 22 \\
\hline Qinghai & 15 & 15 & 283.6 & 50 & 45.8 & 22 \\
\hline Ningxia & 7 & 7 & 664.8 & 50 & 45.8 & 22 \\
\hline Xinjiang & 15 & 15 & 285.3 & 50 & 45.8 & 22 \\
\hline
\end{tabular}


Supplementary Table 13. Reduction of emission intensity for reusing scenario (\%)

\begin{tabular}{|c|c|c|c|}
\hline Region & GHG reduction & $\mathrm{Nr}$ reduction & P reduction \\
\hline Beijing & 7.5 & 25.4 & 25.4 \\
\hline Tianjin & 8.5 & 25.7 & 25.3 \\
\hline Hebei & 11.4 & 26.4 & 25.3 \\
\hline Shanxi & 7.6 & 25.2 & 25.2 \\
\hline Inner Mongolia & 5.9 & 26.2 & 28.7 \\
\hline Liaoning & 7.2 & 23.7 & 26.1 \\
\hline Jilin & 8.1 & 25.4 & 28.7 \\
\hline Heilongjiang & 4.5 & 23.3 & 25.8 \\
\hline Shanghai & 4.9 & 21.8 & 25.3 \\
\hline Jiangsu & 6.8 & 22.6 & 26.0 \\
\hline Zhejiang & 4.8 & 22.5 & 26.8 \\
\hline Anhui & 6.2 & 22.8 & 26.2 \\
\hline Fujian & 4.9 & 21.8 & 25.8 \\
\hline Jiangxi & 2.8 & 21.3 & 25.0 \\
\hline Shandong & 9.6 & 24.2 & 26.9 \\
\hline Henan & 8.6 & 23.4 & 25.9 \\
\hline Hubei & 5.6 & 22.2 & 25.7 \\
\hline Hunan & 3.6 & 23.0 & 27.4 \\
\hline Guangdong & 5.1 & 21.7 & 25.5 \\
\hline Guangxi & 5.1 & 24.0 & 28.7 \\
\hline Hainan & 4.1 & 21.8 & 25.7 \\
\hline Chongqing & 5.5 & 24.4 & 28.7 \\
\hline Sichuan & 6.1 & 24.6 & 29.0 \\
\hline Guizhou & 5.8 & 24.8 & 28.8 \\
\hline Yunnan & 6.2 & 24.7 & 28.7 \\
\hline Shaanxi & 11.5 & 26.2 & 26.0 \\
\hline Gansu & 6.7 & 28.0 & 26.5 \\
\hline Qinghai & 3.6 & 24.8 & 28.7 \\
\hline Ningxia & 8.1 & 31.3 & 34.8 \\
\hline Xinjiang & 6.4 & 25.7 & 28.8 \\
\hline
\end{tabular}




\section{Supplementary Methods}

\subsection{Environmental accounts of agriculture and other sectors}

The accounting of environmental impacts of agriculture is much more complex than other sectors, since the agricultural system contains various subsystems, flows and cycles as well as generates impacts to the water bodies, atmosphere and soil. Subsystems of agriculture include cropland, livestock and aquaculture. We considered nine categories (containing 68 types) of crops products, five categories of aquaculture products (containing 10 types) and seven categories of livestock products.

Obtaining these inventories for Chinese provinces and other regions in the world may vary due to availability of datasets. Except for agriculture sector, we also included other 29 sectors for Mainland China regions and 34 sectors for RoW in concordance with the sector classification scheme in MRIO table. The detailed description of overall environmental accounting is shown in the Supplementary Table 1 .

To compare environmental accounts from our calculations and previous studies, we checked the totaled accounting scope and values from the literature and this is presented in table S1. Water footprint (WF) within the territory was calculated according to Mekonnen and Hoekstra ${ }^{10,11}$ by multiplying parameters by activity data. They reported green, blue and grey WF of one unit of crop and animal products for each state/province of countries. Our calculation considered WF of agriculture, industry and services. For agriculture WF, the part of grain feed in the livestock subsystem was excluded to avoid double counting, since this aspect of WF was accounted in the cropland subsystem. Our results were comparable with those reported by Hoekstra and Mekonnen ${ }^{4}$ and the difference mainly came from production increment and uncertainty of statistical data (see Supplementary Table 2).

For carbon emission inventory, this research broadened the scope of the term 'carbon emission' and considered methane and nitrous oxide emissions from fuel combustion and from agriculture production. For the parameters, we employed the commonly used national standard from IPCC and National Development and Reform Commission $\left(\mathrm{NDRC}^{12}\right)$ where the emission factors and oxidation rate are a little larger than recently reported and applied by Liu et al $(2015)^{6}$. For example, the emission factor of coal combustion is respectively $0.518 \mathrm{tC} / \mathrm{t}$ fuel and $0.491 \sim 0.499 \mathrm{tC} / \mathrm{t}$ fuel in these two set of parameters. This difference in choice of parameters was considered in the uncertainty analysis in the following text. With lower emission factors and revised energy consumption data (so called apparent energy consumption, see references 5 and 6), Shan et al. $(2016)^{5}$ considered carbon dioxide emissions from fuel combustion, while Liu et al. (2015) ${ }^{6}$ accounted for additional emissions from cement production (see Supplementary Table 1). Our primary results were calculated by NDRC emission factors and energy consumption data from Energy Statistical Yearbook ${ }^{13}$, and these optimizations described above were treated as general uncertainty and further assessed with Monte Carlo simulation in the following text.

Reactive nitrogen loss to the environment in this research included fuel combustion in all sectors, cropland, livestock, aquaculture, solid waste and waste water treatment. All the impacts are related to production and could be assigned to one sector in MRIO model, and reactive nitrogen related to human direct emission, grassland and forest were excluded. We made comparisons by summing up corresponding reactive nitrogen loss to atmosphere and waterbody in Figure S5 from Gu et al. 
$(2015)^{7}$ and Figure 2 in Cui et al. (2013) ${ }^{8}$. Due to the high denitrification potential in surface water ${ }^{14}$, Cui et al. reported higher reactive nitrogen accumulation and lower nitrogen $\left(\mathrm{N}_{2}\right)$ emissions. And our results in this research are in consistent with those reported by $\mathrm{Gu}$ et al (2015) (see Supplementary Table 2).

Phosphorus (P) loss to environment include the same subsystems in reactive nitrogen emission accounting. Except $\mathrm{P}$ emissions from production activities flowing to a waterbody, we counted phosphorus accumulated in soil as a kind of resource loss and environmental impact, since more than $85 \%$ of it will be immobilized as "legacy P" and gradually react with elements within the soil, especially in tropical soils ${ }^{15,16}$. Liu et al.(2016) ${ }^{9}$ presented a long time-series of phosphorus flows of China with national level data. We compared accounting from Liu et al. (2016) and found our results are similar but a little larger (see Supplementary Table 1). This may be due to the inconsistency of national statistical data and that from totaling of all provinces.

\subsection{Mapping the grid footprints of food consumption}

Figure 1 in the main text shows the transboundary footprints of food consumption in east and west China. As one province in China can partly belongs to east China and the other part belongs to the west, separated by Hu huanyong line, we divided the footprints of these regions according to the shares of the area attributed to east and west. For example, $99 \%$ of the area of Beijing is assigned to east China, and $99 \%$ of the total footprints of Beijing is assigned to east China.

We accounted per capita footprints of each province in China from the division of MRIO-based footprints and provincial population from the sixth national census in 2010 in China, by assuming a same consumption level within each province. To obtain gridded footprints of food consumption, we multiplied provincial consumption level by the population in each $1 \mathrm{~km}^{2}$ grid (see Supplementary Figure 8). The gridded population here was a combination of mainland China (see ref. ${ }^{17}$, http://www.geodoi.ac.cn/doi.aspx?doi=10.3974/geodb.2014.01.06.v1) and Taiwan province scaled from $1 \mathrm{~km}^{2}$ grid data from Landscan (http://web.ornl.gov/sci/landscan/). With the footprints of each grid, we also standardized these footprints by the min-max scaling method as mentioned in main text.

The definition of the regions mentioned in the main text is described in the Supplementary Table 2.

\subsection{Measuring the environmental stress}

We quantified the environmental and resource stress as described in the main text, and the values of various indicators are listed in Supplementary Table 8.

\subsection{Strategy settings}

Two strategies, reduced food loss and optimized fertilizing, were analyzed in this study to simulate the environmental benefit of mediate actions that could be taken without harm to human health, economic impacts and the farmers' harvest. With the parameters from various literatures (see Supplementary Tables 9 13), we adjusted the entities in MRIO assessment and the results are presented in Supplementary Table 5.

\subsection{Uncertainty analysis}

We determined the standard deviation $(\mathrm{SD}, \sigma)$ of entries in the multi-regional input-output (MRIO) 
table from method proposed in Lenzen et al. $(2010)^{29}$, and that of sectoral environmental accounts by either carrying Monte Carlo simulations or citing previous literatures. Then we quantified the SDs in MRIO calculation where combined environmental accounts and Leontief function by the standard error propagation methods. We chose this method because it will save time in running code compared to the Monte Carlo simulation. We didn't consider uncertainties of environmental accounts of other countries in the world, due to our limited funding and time to collect data country by country.

Firstly, the uncertainty of intermediate flows and final consumptions in Chinese MRIO was estimated from national economic statistics and the census. In 2008 and 2013, China respectively implemented the second and third economy census, and the result showed an overall revision rate (revised amount over total original amount) of about $4.4 \%$ and $3.4 \%$ (ranging from $-2.9 \%$ to $9.0 \%$ within three industries) on total primary GDP statistics ${ }^{30}$. We took these revision rates as the relative standard variations of GDP accounting and the values in China's MRIO table. Uncertainties of total outputs of different industries in the MRIO table were calculated as $r_{x}=m * x^{b}$, where $r_{x}$ is the relative standard deviation (RSD) of a given value $x$, and $\mathrm{m}$ and $\mathrm{b}$ are two parameters in year 2010 determined by results from economy census in 2008 and 2013. We refer to Lenzen et al. (2010) ${ }^{29}$ paper for more detailed inductions of this method.

Secondly, we determined the SDs of environmental account for WF, CF, VNF and VPF, separately. For WF calculations, we recognized that the livestock subsystem took less than $4 \%$ of the total agriculture WF if we excluded that of grain feed to prevent double counting. Thus, we took WF uncertainty from cropland as a proxy of the total agriculture WF uncertainty. WF related to per unit mass of crop production was calculated by dividing water use by crop yield in a certain area, and the value depended on precipitation, soil water content, reference evapotranspiration, runoff and other variables. Zhuo et al. ${ }^{31}$ evaluated the uncertainties of WF from input of key variables mentioned above for the main crop types in Chinese Yellow River Basin and found around 20 30\% ( $2 \sigma$ gave the $95 \%$ confidence level) due to errors from key variables.

There are few reports on CF accounting uncertainties for China, thus we used MCS method to quantify the relative standard deviation by collecting localized emission factors and statistical data. Recent research ${ }^{6}$ showed that emission factors recommended by the Intergovernmental Panel on Climate Change (IPCC) were much larger than those actually measured in China, and so we presented uncertainty range of 24 possible carbon dioxide inventories of China. However, we also considered greenhouse gas emission from rice paddy (methane), cropland and livestock (methane and nitrous oxide), and focus more on uncertainties caused by emission factors reported domestically. Within China, the commonly used emission factors came from China's National Communication $^{32}$ (national GHG inventory which is reported to United Nations), National Development and Reform Commission (NDRC) ${ }^{12}$ and reported in literature ${ }^{6}$. For energy GHG emissions, we took the differences between NDRC and Liu et al. $(2016)^{6}$ and that between summary of provincial energy consumption and national energy statistics as the uncertainty ranges $(2 \sigma)$. Uncertainties of emission factors for agriculture were taken from the national GHG inventory studies $^{33}$, while all other Chinese statistical data was taken a $10 \%$ interval. A population of 10000 samples were performed and the SDs were reported at a $95 \%$ confidence interval.

For VNF, the uncertainties will propagate through the process of the modeling of material flow and were thoroughly considered in the literature ${ }^{8,34,35}$. Reactive nitrogen estimations in this study also followed a cascade model by considering material flow process among different subsystems. Three 
main sources of uncertainty examined would include structure of the model, parameter values and propagation of prediction errors. Uncertainty of reactive nitrogen emissions in this study was taken from Shi et al. ${ }^{34}$ and was valued as $8.4 \%(2 \sigma)$ for the range of total emissions.

Quantification of VPF also followed the process of material flow among subsystems and the uncertainty could be determined by the same method as that of VNF. Liu et al. (2016) ${ }^{9}$ recognized sources of uncertainty as various parameters taken from different researches and the statistical data and quantified the uncertainty range, therefore we took a $26.4 \%(2 \sigma)$ interval of phosphorus runoff in Liu et al. $(2016)^{9}$ as the uncertainty in our phosphorus emission inventory.

Thirdly, we quantified the uncertainty range in the calculation of footprints in MRIO model with the standard error propagation methods based on this analytical approach. Compared with sampling approach, this method will largely save time for calculation but basically provide the same results ${ }^{36}$. With the basic rules shown in function (3) in the main text and data preparation stated above, we can should further determine the algorithm of variables such as the SD of environmental intensity $\sigma_{e_{i}}$ and others. As $e=E \hat{X}^{-1}$ was derived from the division of two random variables, with the laws of error propagation, we had

$\frac{\left(\sigma_{e_{i}}\right)^{2}}{e_{i}{ }^{2}}=\frac{\left(\sigma_{E_{i}}\right)^{2}}{E_{i}{ }^{2}}+\frac{\left(\sigma_{X_{i}}\right)^{2}}{X_{i}{ }^{2}}$,

so the $\mathrm{SD}$ of environmental intensity was calculated as

$\sigma_{e_{i}}=\sqrt{e_{i}^{2}\left[\frac{\left(\sigma_{E_{i}}\right)^{2}}{E_{i}{ }^{2}}+\frac{\left(\sigma_{X_{i}}\right)^{2}}{X_{i}{ }^{2}}\right]}$.

Then we calculated the second item in function (3), namely $\left(\frac{\partial F}{\partial L_{i j}}\right)^{2} \sigma_{L_{i j}}$. As $F=e L y$, according to function (47) in Heijungs and Lenzen ${ }^{37}$ and function (S8) in Oita et al. ${ }^{38}$, we had

$$
\begin{aligned}
\sum_{i=1}^{n} \sum_{j=1}^{n}\left(\frac{\partial F}{\partial L_{i j}}\right)^{2} \sigma_{L_{i j}} & =\sum_{i=1}^{n} \sum_{j=1}^{n}\left[(q L)_{i}(L y)_{j} \ln (10) A_{i j}\right]^{2}\left(\sigma_{\log _{10} z_{i j}}\right)^{2} \\
& =\sum_{i=1}^{n} \sum_{j=1}^{n}\left[\frac{(q L)_{i}(L y)_{j} A_{i j}}{Z_{i j}}\right]^{2}\left(\sigma_{\log _{10} Z_{i j}}\right)^{2},
\end{aligned}
$$

Where $A_{i j}$ and $Z_{i j}$ respectively denote the direct requirement matrix and intermediate flow in the MRIO table. We refer to Heijungs and Lenzen ${ }^{37}$, and Oita et al. ${ }^{38}$ for detailed deduction.

With the laws of error propagation, we can get SD of final consumption $\left(\sigma_{y_{j}}\right)$ from $m$ items of final consumption,

$\sigma_{y_{j}}=\sqrt{\sum_{k=1}^{m}\left(\sigma_{y_{j k}}\right)^{2}}$.

The results of uncertainties show that smaller footprints usually show larger relative standard deviation (RSD), while larger footprints show smaller RSD. The above figure present RSDs of footprints of three urban regions coming from different regions, most of the footprints have a RSD less than $10 \%$. Except for some smaller footprints, the overall footprints from our model have a high reliability.

Moreover, more errors that have been recognized previously were not simulated in this study: these included as errors from sectoral, spatial aggregation and RAS method in MRIO estimation, and data taken from various sources. We refer interested readers to the literature $\mathrm{e}^{6,8,9,29,31,34,39-43}$ for more detailed treatment of uncertainties from different certain aspects. 


\section{Supplementary References:}

Liu, W., Tang, Z., Chen, J. \& Yang, B. China's inter-regional input-output table of 30 provinces and cities in 2010 (China Statistic Press, 2014).

NBSC. China Statistical Yearbook. (China Statistics Press, 2016).

NBSC. Input-Output Table of China 2012. (China Statistics Press, 2016). Hoekstra, A. Y. \& Mekonnen, M. M. The water footprint of humanity. P Natl Acad Sci USA 109, 3232-3237 (2012).

Shan, Y. et al. New provincial $\mathrm{CO}_{2}$ emission inventories in China based on apparent energy consumption data and updated emission factors. Applied Energy (2016).

Liu, Z. et al. Reduced carbon emission estimates from fossil fuel combustion and cement production in China. Nature 524, 335-338 (2015).

Gu, B., Ju, X., Chang, J., Ge, Y. \& Vitousek, P. M. Integrated reactive nitrogen budgets and future trends in China. Proceedings of the National Academy of Sciences 112, 8792-8797 (2015).

Cui, S., Shi, Y., Groffman, P. M., Schlesinger, W. H. \& Zhu, Y.-G. Centennial-scale analysis of the creation and fate of reactive nitrogen in China (1910-2010). Proceedings of the National Academy of Sciences 110, 2052-2057 (2013).

Liu, X. et al. Intensification of phosphorus cycling in China since the 1600s. Proceedings of the National Academy of Sciences 113, 2609-2614 (2016).

Mekonnen, M. M. \& Hoekstra, A. Y.

(UNESCO-IHE Institute for Water Education, Delft, the Netherlands, 2010).

1

Mekonnen, M. M. \& Hoekstra, A. Y. (UNESCO-IHE Institute for Water Education, Delft, the Netherlands, 2010).

NDRC. Guidelines for China's Provincial GHG Emission Inventories (NDRC, 2012).

NBS. China Energy Statistical Yearbook. (China Statistics Press, 2011).

Zhao, Y. et al. Nitrogen removal capacity of the river network in a high nitrogen loading region. Environmental science \& technology 49, 1427-1435 (2015).

5 Batjes, N. Global distribution of soil phosphorus retention potential. (ISRIC-World Soil Information, 2011).

6 Palm, C., Sanchez, P., Ahamed, S. \& Awiti, A. Soils: A contemporary perspective. Annu. Rev. Environ. Resour. 32, 99-129 (2007).

$17 \mathrm{Fu}$, J., Jiang, D. \& Huang, Y. Dataset of $1 \mathrm{~km} 2$ Grid Population distribution of China (in Chinese). ACTA GEOGRAPHICA SINICA 69, 41-44 (2014).

Liu, G. Food Losses and Food Waste in China. OECD Food, Agriculture and Fisheries Papers No. 66, OECD Publishing (2014).

Liu, J., Lundqvist, J., Weinberg, J. \& Gustafsson, J. Food losses and waste in China and their implication for water and land. Environmental science \& technology 47, 10137-10144 (2013).

Zhang, S. et al. Overcoming nitrogen fertilizer over-use through technical and advisory approaches: A case study from Shaanxi Province, northwest China. Agriculture, Ecosystems \& Environment 209, 89-99 (2015).

Ju, X.-T. et al. Reducing environmental risk by improving $\mathrm{N}$ management in intensive

Chinese agricultural systems. Proceedings of the National Academy of Sciences 106, 3041-3046 (2009). 
Chen, X., Zhang, F. \& Jiang, R. The technique system of soil/plant quick test and recommend fertilizer. Integrated management of nutrient resources. China Agriculture Press, Beijing (2003).

Zhang, W. et al. Efficiency, economics, and environmental implications of phosphorus resource use and the fertilizer industry in China. Nutrient Cycling in Agroecosystems $\mathbf{8 0}$, 131-144 (2008).

Lu, F. et al. Soil carbon sequestrations by nitrogen fertilizer application, straw return and no-tillage in China's cropland. Global Change Biology 15, 281-305 (2009).

Liu, X., Xu, J., Wang, F., Zhang, F. \& Ma, W. The distribution of nitrogen resources of animal manure in China. Journal of Agricultural University of Hebei 28, 27-32 (2005).

Xu, J., Liu, X., Wang, F., Zhang, F. \& Ma, W. The distribution of phosphorus resources and utilization of animal manure in China. Journal of Agricultural University of Hebei 28, 5-9 (2005).

27 Cordell, D., Rosemarin, A., Schröder, J. \& Smit, A. Towards global phosphorus security: A systems framework for phosphorus recovery and reuse options. Chemosphere 84, 747758 (2011).

Wang, F., Zhang, F. \& Ma, W. The present situation and the countermeasures of agricultural non-point source pollution in China (Report to the Department of Agriculture). (2007).

Lenzen, M., Wood, R. \& Wiedmann, T. Uncertainty analysis for multi-region input-output models-a case study of the UK's carbon footprint. Economic Systems Research 22, 43-63 (2010).

Xu, C. in Comparative Studies Vol. 2 (ed Jinglian Wu) (China CITIC Press, 2015).

Zhuo, L., Mekonnen, M. \& Hoekstra, A. Sensitivity and uncertainty in crop water footprint accounting: a case study for the Yellow River basin. Hydrology and earth system sciences 18, 2219-2234 (2014).

NDRC. Second National Communication on Climate Change of the People's Republic of China (NDRC, Department of Climate Change) (2012).

$\mathrm{Su}, \mathrm{W}$. The People's Republic of China National Greenhouse Gas Inventory 2005. (China Environmental Press, 2014).

Shi, Y., Cui, S., Ju, X., Cai, Z. \& Zhu, Y.-G. Impacts of reactive nitrogen on climate change in China. Scientific reports 5, 8118 (2015).

Cui, S. et al. A hybrid method for quantifying China's nitrogen footprint during urbanisation from 1990 to 2009. Environment International (2016).

Heijungs, R., Suh, S. \& Kleijn, R. Numerical Approaches to Life Cycle Interpretation-The case of the Ecoinvent'96 database (10 pp). The International Journal of Life Cycle Assessment 10, 103-112 (2005).

37 Heijungs, R. \& Lenzen, M. Error propagation methods for LCA -a comparison. The International Journal of Life Cycle Assessment 19, 1445-1461 (2014).

Oita, A. et al. Substantial nitrogen pollution embedded in international trade. Nature Geoscience 9, 111 (2016).

39 Su, B. \& Ang, B. W. Input-output analysis of $\mathrm{CO}_{2}$ emissions embodied in trade: competitive versus non-competitive imports. Energy Policy 56, 83-87 (2013).

40 Su, B., Huang, H., Ang, B. \& Zhou, P. Input-output analysis of $\mathrm{CO}_{2}$ emissions embodied in 
trade: the effects of sector aggregation. Energy Economics 32, 166-175 (2010).

41 Su, B. \& Ang, B. W. Input-output analysis of $\mathrm{CO}_{2}$ emissions embodied in trade: the effects of spatial aggregation. Ecological Economics 70, 10-18 (2010).

42 Inomata, S., and Anne O. Comparative evaluation of MRIO databases. Economic Systems Research 26, 239-244 (2014).

43 Lenzen, M., Pade, L.-L. \& Munksgaard, J. CO2 multipliers in multi-region input-output models. Economic Systems Research 16, 391-412 (2004). 